\title{
Photoacoustic imaging using genetically encoded reporters: a review
}

Joanna Brunker

Junjie Yao

Jan Laufer

Sarah E. Bohndiek 


\title{
Photoacoustic imaging using genetically encoded reporters: a review
}

\author{
Joanna Brunker, ${ }^{a}$ Junjie Yao,, Jan Laufer, ${ }^{\mathrm{c}}$ and Sarah E. Bohndiek ${ }^{\mathrm{a}, \boldsymbol{}}$ \\ aUniversity of Cambridge, Cancer Research UK Cambridge Institute and Department of Physics, Cambridge, United Kingdom \\ ${ }^{b}$ Duke University, Photoacoustic Imaging Lab, Department of Biomedical Engineering, Durham, North Carolina, United States \\ ${ }^{c}$ Martin-Luther-Universität Halle-Wittenberg, Institut für Physik, Halle (Saale), Germany
}

\begin{abstract}
Genetically encoded contrast in photoacoustic imaging (PAI) is complementary to the intrinsic contrast provided by endogenous absorbing chromophores such as hemoglobin. The use of reporter genes expressing absorbing proteins opens the possibility of visualizing dynamic cellular and molecular processes. This is an enticing prospect but brings with it challenges and limitations associated with generating and detecting different types of reporters. The purpose of this review is to compare existing PAI reporters and signal detection strategies, thereby offering a practical guide, particularly for the nonbiologist, to choosing the most appropriate reporter for maximum sensitivity in the biological and technological system of interest. ๑ 2017 Society of Photo-Optical Instrumentation Engineers (SPIE) [DOI: 10.1117/1.JBO.22.7.070901]
\end{abstract}

Keywords: genetic reporters; in vivo; photoacoustics.

Paper 170235VR received Apr. 12, 2017; accepted for publication Jun. 12, 2017; published online Jul. 17, 2017.

\section{Introduction}

Combining optical excitation and acoustic detection, photoacoustic imaging (PAI) combines the high contrast available from optical imaging with the spatiotemporal resolution of ultrasound imaging. Conventional reporter genes (RGs) for optical imaging generate diffuse bioluminescence or fluorescence signals, which can be imaged with spatial resolutions that are typically comparable to, or worse than, the imaging depth. ${ }^{1,2}$ PAI transcends the depth limitations of optical imaging techniques that employ ballistic photons (Fig. 1) because acoustic waves are scattered far less than photons in tissue. The contrast in PAI arises due to optical absorption, for example by endogenous chromophores such as hemoglobin and melanin. However, to directly visualize most cellular and molecular processes, the image contrast must be provided by exogenous agents. ${ }^{3}$ A RG is an exogenous segment of DNA that encodes a protein product that, once expressed, can be visualized, either directly or indirectly, using imaging or chemical analysis. The application of RGs in optical imaging, magnetic resonance imaging (MRI), and nuclear medicine has been reviewed elsewhere. ${ }^{4,5}$ PAI RGs have been reviewed from the perspective of PAI technology; ${ }^{6}$ here, we provide a complementary practical guide to PAI RGs for the physical scientist, who may be unfamiliar with the biological background and the fundamental limitations. We highlight, in particular, the challenges of: biological RG production and characteristics (Sec. 3); spectral unmixing of the reporter signal (Sec. 4); and detection sensitivity (Sec. 5).

\section{Principles of Photoacoustic Imaging}

PAI starts with the excitation of molecules in biological tissues following absorption of photons from pulsed or modulated continuous-wave light sources. The excited molecules release the absorbed photon energy through nonradiative relaxation,

\footnotetext{
*Address all correspondence to: Sarah E. Bohndiek, E-mail: seb53@ cam.ac.uk
}

inducing a small local temperature rise. This leads to a local pressure increase, which then relaxes resulting in the propagation of ultrasound (photoacoustic) waves throughout the tissue; these waves can be detected by ultrasonic transducers at the surface of the tissue.

PA image reconstruction essentially converts the detected time-resolved ultrasound signals back to the optical absorption distribution in space, a procedure similar to the Global Positioning System. This is a two-step problem involving first, reconstruction of the initial acoustic pressure distribution (the acoustic inverse problem) and second, recovery of the optical absorption distribution (the optical inverse problem). Various reconstruction approaches ${ }^{7}$ have been employed from the simplest and fastest method of back-projection, ${ }^{8,9}$ to more complex approaches that can account for arbitrary scanning geometries, such as model-based linear inversion, ${ }^{10}$ iterative methods, ${ }^{11}$ time-reversal, and fast Fourier transform-based methods. ${ }^{12,13}$ Solution of the optical inverse problem for accurate quantification of the optical absorption distribution remains challenging ${ }^{14}$ but is essential for quantitative molecular imaging (discussed further in Sec. 4).

PAI is a highly scalable imaging modality, achieving different spatial resolutions and imaging depths based on different configurations of: light sources; ultrasonic detection systems; and scanning mechanisms. ${ }^{15,16}$ Based on the image formation method, PAI can be roughly classified into: photoacoustic microscopy (PAM) with single-element ultrasonic detection and direct image formation; or photoacoustic computed tomography (PACT) with multielement ultrasonic detection and inverse image reconstruction (see Fig. 2). Both image resolution and tissue attenuation scale with increasing ultrasound detection frequency. In general, PAM, with high-frequency ultrasound detection at tens of $\mathrm{MHz}$, provides micron-resolution images with penetration depths up to several millimeters, whereas PACT, with low-frequency ultrasound detection at a few MHz, is capable of deep-tissue imaging with centimeter penetration and submillimeter resolutions. 


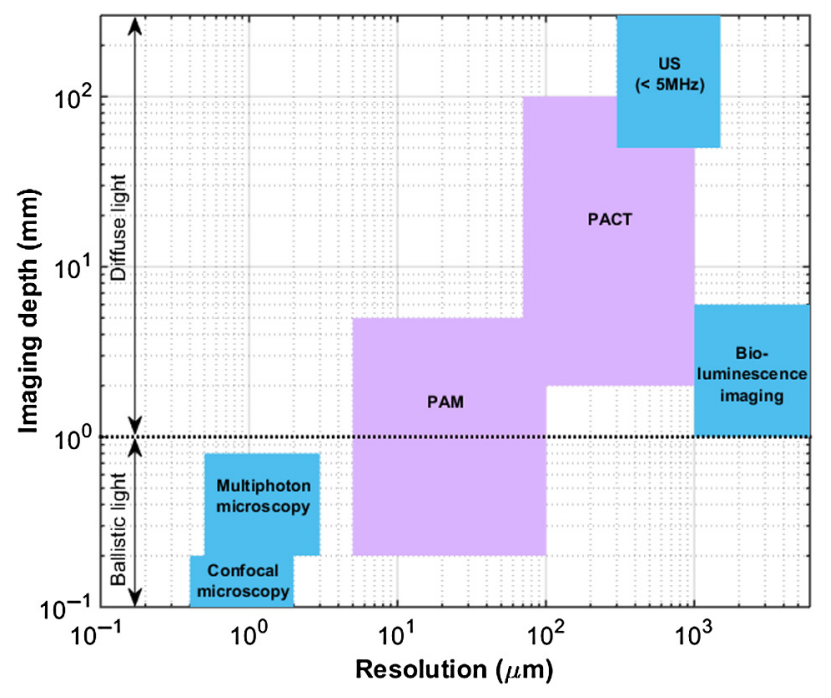

Fig. 1 Comparison of the performance of PAI with that of optical and ultrasonic modalities in terms of their imaging depth and spatial resolution. PAM, photoacoustic microscopy; PACT, photoacoustic computed tomography; US, ultrasound imaging.

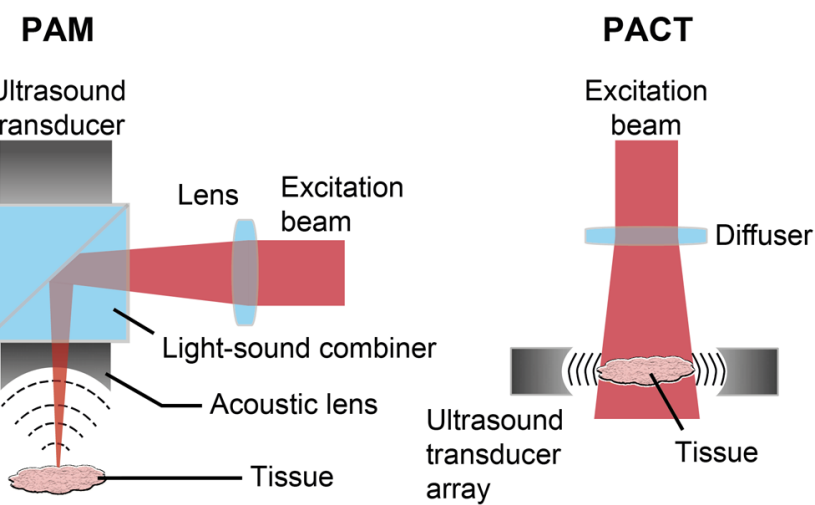

Fig. 2 Schematics of representative PA imaging implementations, illustrating PAM and PACT. In PAM, the spatial resolution can be defined optically (OR-PAM) or acoustically (AR-PAM). Both OR-PAM and AR-PAM use raster scanning of a single-element ultrasound transducer to achieve volumetric imaging, shown here in reflection mode. PACT uses an ultrasound transducer array to detect PA waves emitted at multiple view angles simultaneously.

\section{Genetic Reporters used in Photoacoustic Imaging}

\subsection{Production of Cells Expressing Reporter Proteins}

The production of genetically modified mammalian cells involves transfection, which is the general term describing a procedure for introducing foreign nucleic acids, such as those constituting a RG, into eukaryotic cells. Transfection methods can be broadly categorized into those that are transient and others that are stable, illustrated in Figs. 3(a) and 3(b) respectively. Transient transfection (TT) is relatively straightforward, involving the introduction of DNA into cells along with an agent that promotes its uptake. However, since the foreign DNA is not integrated into the host genome, the sequence is gradually lost after repeated cell divisions leading to gene expression that lasts only a few days. Alternatively, stable transfection entails integration of the foreign genetic material into the host genome giving rise to long-term expression of the sequence of interest as it is propagated in subsequent cell generations.

Methods for TT are generally physical or chemical. ${ }^{17}$ The most commonly used physical method is electroporation, which can rapidly transfect large numbers of cells, but only after an experimentally demanding determination of the optimum conditions. PAI studies to date have predominantly used chemical methods for TT. These methods include use of a cationic liposome, ${ }^{18}$ a cationic lipid, ${ }^{19}$ or calcium phosphate,$^{20}$ and are widely available but may vary in their toxicity and efficiency according to the cell type. The RG can be coexpressed with an antibiotic resistance gene and/or a fluorescent marker such as EGFP or an inert membrane tag such as the truncated nerve growth factor receptor (dNGFR). These enable successfully transfected cells to be selected by their survival in the presence of antibiotic, and also the reporter protein expression level to be identified using flow cytometry of the coexpressed marker.

Stable transfection typically involves virus-mediated gene delivery, ${ }^{21}$ which is also known as transduction. The first step in the transduction process is to transfect three or four plasmids - one envelope vector, one or two packaging vectors, and the transfer vector containing the RG-into packaging cells such as human embryonic kidney 293T cells (HEK293T). The virus is then harvested from these cells and used to infect the target cells, resulting in incorporation of the RG sequence into the host DNA. Photoacoustic RG studies have employed common viral vectors including lentiviruses ${ }^{20,22}$ and retroviruses. ${ }^{23,24}$ Lentiviruses are more versatile since they transduce nondividing cells, unlike retroviruses, which require replicating cells for transduction. A third viral vector system that has been used is the vaccinia virus, ${ }^{25}$ but this is limited to transient protein production since the virus replicates in the cytoplasm outside the nucleus and the infected cells only survive for one or two days.

\subsection{Classes of Reporters}

PAI RGs can be classified as indirect (enzymatic, which result in nonfluorescent proteins, Sec. 3.3) or direct (fluorescent and nonfluorescent proteins, Sec. 3.4). These classes are shown in Fig. 4, and their spectra are given in Fig. 5. Enzymatic RGs provide inherent signal amplification, as a single enzyme can create multiple molecules of absorbing product, whereas direct RGs provide 1:1 stoichiometric mapping between the amount of detected protein and the level of RG expression. Table 1 makes further comparisons between the different classes of reporters. The ideal reporter should be: specific to the biological process of interest; exhibit an absorption maximum in the tissue optical window for deep in vivo imaging; be nontoxic to the cell; and avoid photobleaching. The reviewed RGs, including optical properties and demonstrated applications, are discussed in the following two sections and summarized in Table 2.

\subsection{Enzymatic Reporters}

Three enzymatically generated reporters have been introduced for PAI: (1) 5,5'-dibromo-4,4'-dichloroindigo, a blue precipitate produced via the enzyme $\beta$-galactosidase (encoded by the lacZ gene), which metabolizes X-gal; (2) eumelanin, a brown pigment produced through action of the tyrosinase enzyme on 
(a)

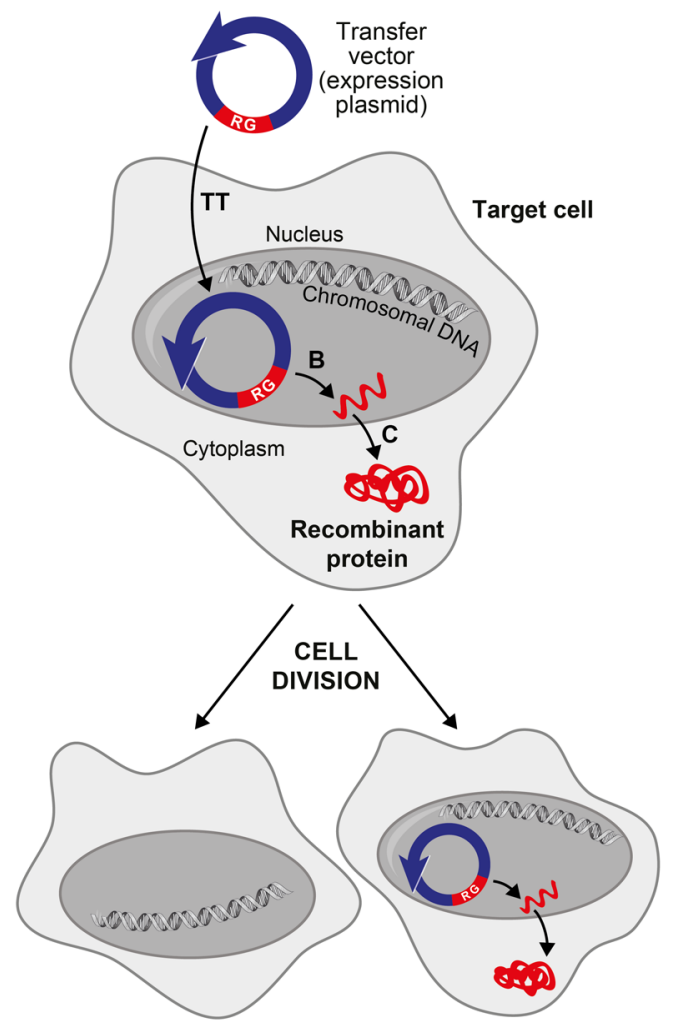

(b)

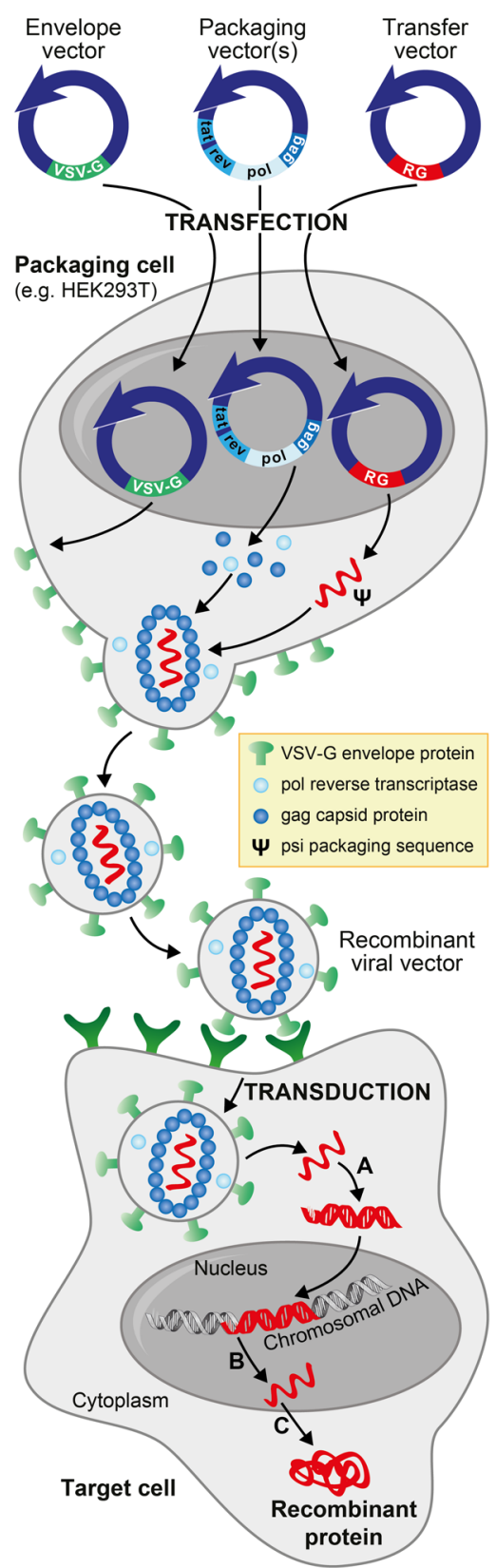

Fig. 3 Schematics illustrating (a) TT and (b) stable transfection of an RG into a mammalian cell. The gene must first be introduced into the target cell (for example, by electroporation), and subsequently into the nucleus: these two steps represent TT. The recombinant protein is expressed but after cell division the DNA is only passed on to one of the daughter cells, resulting in the dilution of the RG with subsequent divisions. Stable expression over successive cell generations is achieved by integration of the RG into the target cell's chromosomal DNA (b). This process typically involves lentiviral or retroviral vectors, which are produced by packaging cells such as HEK293T. The viral genes are expressed by several plasmids (also called vectors): (i) an envelope vector encoding the vesicular stomatitis virus (VSV-G) envelope gene; (ii) one or two packaging vector(s) encoding the pol, gag, rev, and tat viral genes; and (iii) a viral expression plasmid (transfer vector) containing the psi $\Psi$ packaging sequence and the RG. Approximately 2 days after transfection of HEK293T cells, the cell supernatant contains recombinant viral vectors, which can be used to transduce the target cells. Once in the target cells, the viral RNA is reverse-transcribed (A), imported into the nucleus and stably integrated into the host genome. The RG is transcribed into mRNA (B) and then translated into the reporter protein (C). Expression of the recombinant protein can be detected 1 or 2 days after integration of the viral RNA.

tyrosine; and (3) violacein, a violet pigment produced from L-Tryptophan via the action of a five-enzyme operon.

LacZ. LacZ is the earliest example of a PAI reporter. ${ }^{44}$ It expresses $\beta$-galactosidase, an Escherichia coli enzyme involved in the metabolism of lactose. Injection of X-gal, a colorless analogue of lactose, leads to formation of a blue product $\left(5,5^{\prime}\right.$ dibromo-4,4'-dichloroindigo) after cleavage by $\beta$-galactosidase [Fig. 6(a)]. The blue product is strongly absorbing between 
(a)

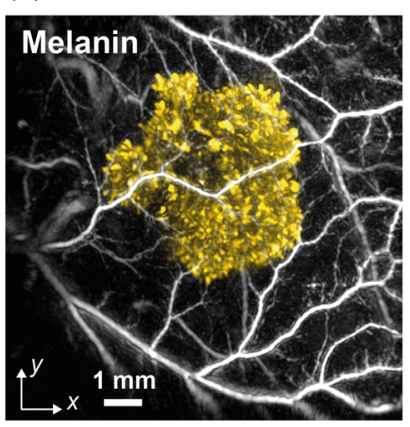

(b)

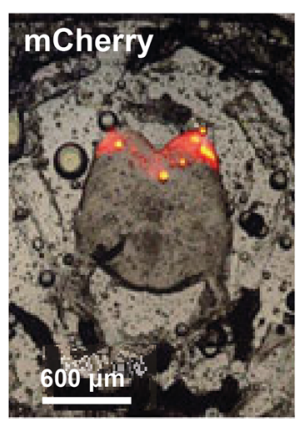

DIRECT

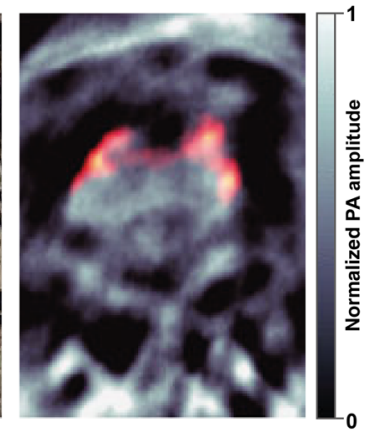

(c)

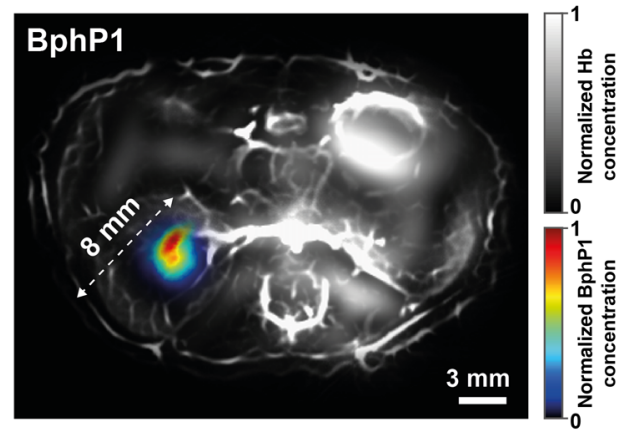

Fig. 4 Selected studies of PA RG imaging. (a) PA image of a tyrosinase-expressing tumor (shown in yellow) after subcutaneous injection into the flank of a nude mouse. ${ }^{24}$ The surrounding blood vessels are shown in gray. (b) Left: fluorescent histology of the brain of a transgenic zebrafish expressing mCherry (shown in color); right: corresponding PA image ${ }^{26}$ (c) PA image of a kidney tumor expressing reversibly switchable nonfluorescent bacterial phytochrome BphP1. ${ }^{19}$ The tumor is shown in color, and the background blood-rich organs are shown in gray. $\mathrm{Hb}$, hemoglobin.

600 and $700 \mathrm{~nm}$. Dual-wavelength $\mathrm{PAM}^{42,45}$ and $\mathrm{PACT}^{45}$ have been used to detect gliosarcoma cells expressing the lacZ gene under the rat scalp; however, $\mathrm{X}$-gal requires local administration as systemic delivery is inefficient, ${ }^{44}$ which has discouraged further PAI studies with the lacZ gene.

Tyrosinase. Tyrosinase oxidizes tyrosine to dopaquinone, which then undergoes several reactions that lead to melanin pigments [Fig. 6(b)] ${ }^{46}$ including eumelanin and pheomelanin. Eumelanin exhibits a broad extinction coefficient spectrum (similar to that of pheomelanin), with absorption extending into the near-infrared, enabling it to be detected above the background hemoglobin signal at wavelengths between 600 and $700 \mathrm{~nm} .{ }^{18,24,47}$ Paproski et al. ${ }^{18}$ were the first to develop tyrosinase as an RG for PAI. They subsequently developed an inducible system, in which tyrosinase expression was triggered by doxycycline administered in the animals' drinking water. ${ }^{47}$ The tyrosinase reporter has also shown multimodal imaging potential $^{48}$ as eumelanin can bind heavy metal ions $\left(\mathrm{Fe}^{3+}\right)$ detectable with MRI, and a melanin-targeted ${ }^{18} \mathrm{~F}-\mathrm{P} 3 \mathrm{BZA}$ probe for positron emission tomography. Stritzker et al. ${ }^{25}$ also explored multimodal tyrosinase imaging by TT of tumor bearing mice with a vaccinia virus encoding tyrosinase. This approach offers clinical relevance but prevents longitudinal studies. To maintain tyrosinase expression in subsequent generations of cells requires modification of the cell genome, which, although ethically unapproved for clinical studies, does allow longitudinal animal imaging. Jathoul et al. ${ }^{24}$ achieved this using stable retroviral transduction, which enabled tumor growth to be monitored over periods up to 52 days, with apparently little effect on cell viability [Fig. 4(a)].

Violacein. The deep violet chromophore violacein is enzymatically generated [Fig. 6(c)] from the sole precursor tryptophan by five enzymes (VioA-E) that were originally cloned from Chromobacterium violaceum. Violacein has a strong absorption peak around $575 \mathrm{~nm}$, enabling differential detection through dual-wavelength excitation at 490 and $650 \mathrm{~nm}$. However, while tyrosinase and lacZ have been expressed in mammalian cells, the violacein operon has so far only been expressed in bacteria. ${ }^{50}$ Violacein may, therefore, find application for in vivo imaging of bacterial infections or theranostic applications, but concerns remain about the toxicity to mammalian cells.

\subsection{Nonenzymatic Reporters: Fluorescent, Nonfluorescent, and Photoswitchable Proteins}

Nonenzymatic reporters can be classified by their optical properties and according to their natural origins, such as marine creatures and bacteria [Figs. 5(a)-5(c), Fig. 7]. In general, nonfluorescent proteins are preferable since they exhibit higher photoacoustic generation efficiency and significantly higher photostability (Fig. 8).

\subsubsection{Fluorescent proteins}

Since the discovery of green fluorescent protein (GFP), fluorescent proteins have been widely applied in biomedical studies, mostly imaged by fluorescence microscopy. ${ }^{27,51,52}$ GFP-like proteins are derived from anthozoa (such as sea anemones and corals) or scyphozoa (jellyfish) and have a common structure consisting of a $\beta$-barrel scaffold containing the chromophore, which is formed by folding of the polypeptide chain. ${ }^{53}$ Within the barrel the chromophore is protected from the outside environment, making it possible to genetically engineer the chromophore microenvironment, for example, to produce enhanced brightness (EGFP) ${ }^{54}$ or spectral modifications. Razansky et al. ${ }^{26}$ reported the first demonstration of PAI using EGFP and mCherry in fruit fly and adult zebrafish [Fig. 4(b)]. The absorption peaks of even the furthest red-shifted GF homologues ${ }^{35,55}$ are generally restricted to the visible wavelength region, making them well suited to PAM but less applicable for PACT deep in vivo imaging [Fig. 5(a)].

In recent years, several near-infrared fluorescent proteins (IFPs) have been derived from bacterial phytochrome photoreceptors (BphPs) ${ }^{56}$ These are targeted toward deep tissue in vivo imaging, exploiting the optical window where endogenous chromophores have relatively low absorption between 620 and $950 \mathrm{~nm}$ [Fig. 5(d) and 5(e)]. BphPs incorporate a far-red absorbing bilin, biliverdin (BV), as their chromophore, which is ubiquitous in most eukaryotic organisms. The overall structure of the BphP photosensory module consists of two or three protein domains linked by $\alpha$-helices (Fig. 9), which may exist as a monomer, dimer, or oligomer. As with GFP-like proteins, monomers are preferable and several monomeric IFPs have been developed. ${ }^{57-59}$ Compared to GFP-like proteins, BphPs 


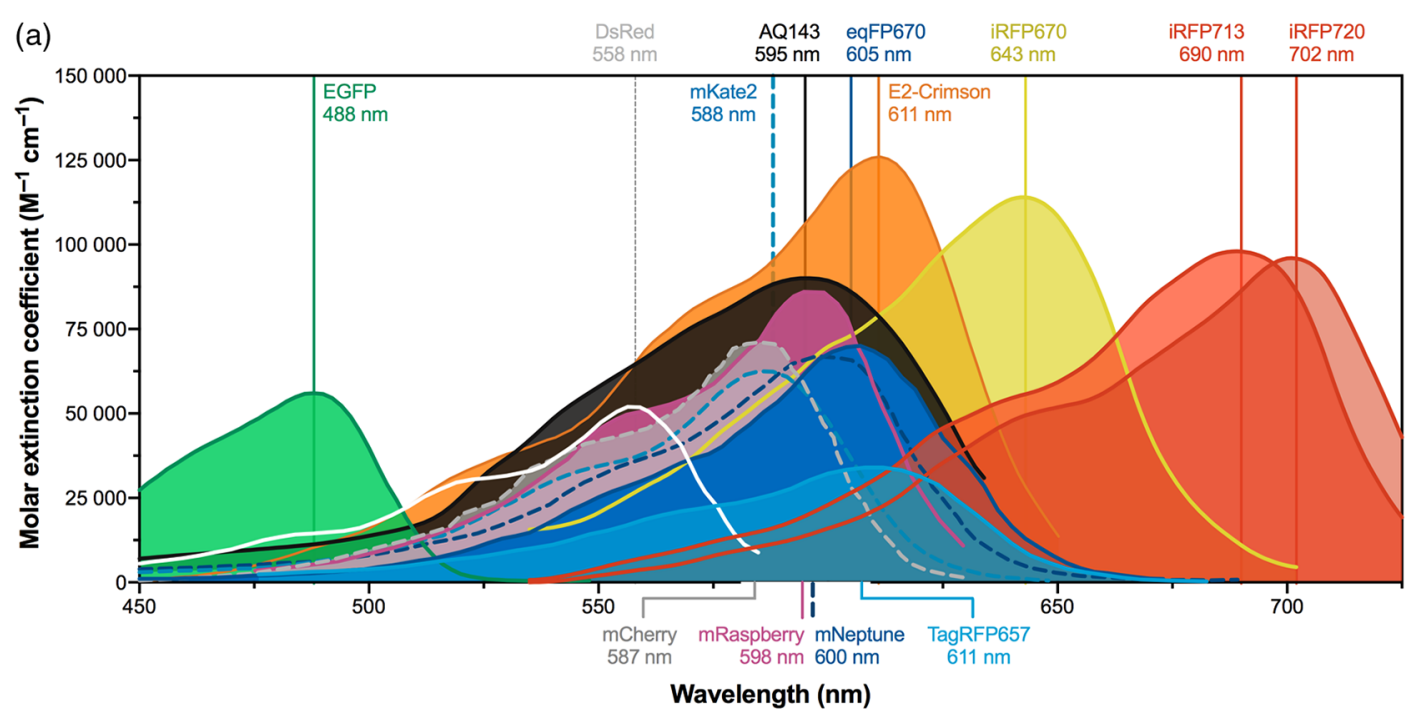

(b)

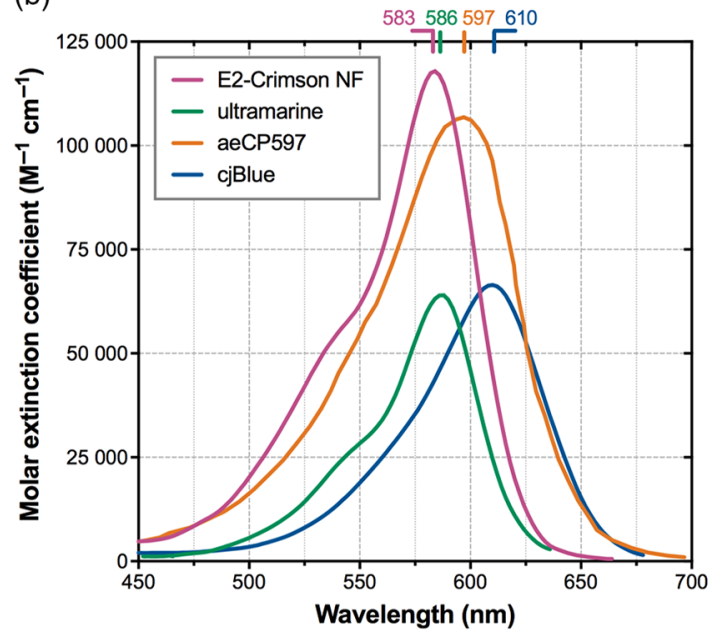

(d)

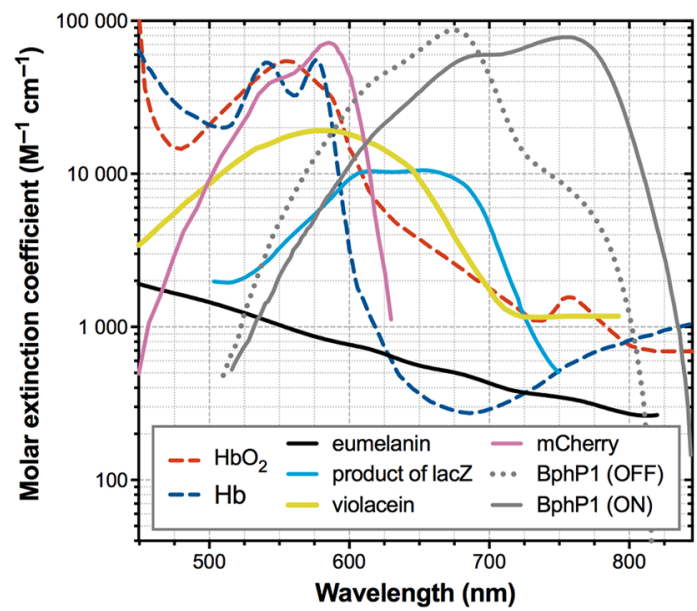

(c)

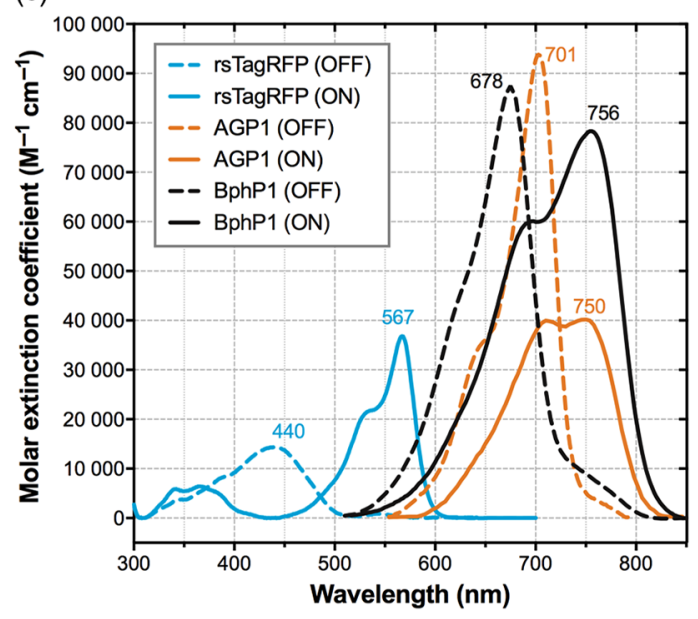

(e)

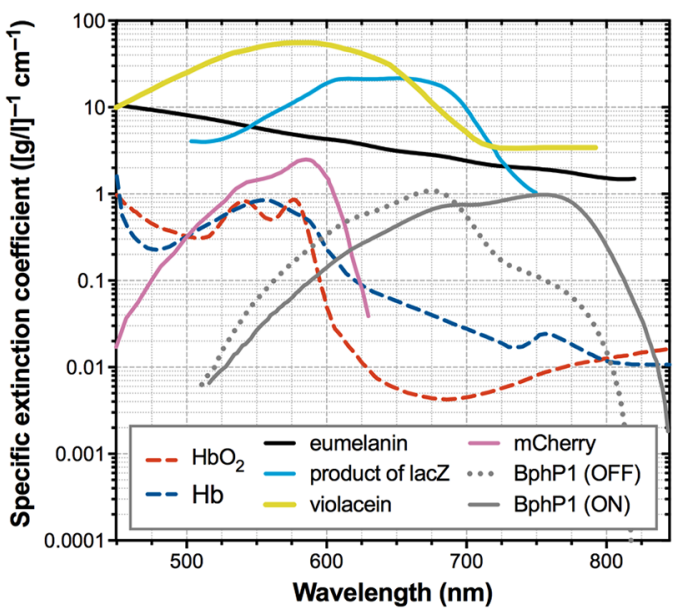

Fig. 5 Summary of all spectra for genetically encoded reporter proteins demonstrated for PAI. (a) Fluorescent proteins: EGFP, ${ }^{26,27}$ DsRed, ${ }^{28}$ mCherry, ${ }^{29}$ mKate2, ${ }^{30}$ AQ143, ${ }^{31}$ mRaspberry, ${ }^{32}$ mNeptune, ${ }^{33}$ eqFP670, ${ }^{34}$ E2-Crimson, ${ }^{35}$ TagRFP657, ${ }^{36}$ iRFP670, ${ }^{37}$ iRFP713 (iRFP), ${ }^{37}$ iRFP720. ${ }^{37}$ (b) Chromoproteins: E2-Crimson NF; ${ }^{23}$ ultramarine; ${ }^{38}$ aeCP597; ${ }^{31}$ cjBlue. ${ }^{39}$ (c) Photoswitchable proteins: rsTagRFP; ${ }^{19}$ AGP $1 ; 22$ BphP1. ${ }^{19}$ (d) Molar extinction coefficients and (e) specific extinction coefficients are compared to oxyhemoglobin $\left(\mathrm{HbO}_{2}, 150 \mathrm{gl}^{-1}\right.$, red dashed line $\left.\mathrm{H}^{40}\right)$ and deoxyhemoglobin $\left(\mathrm{Hb}, 150 \mathrm{gl}^{-1}\right.$, blue dashed line, $\left.{ }^{40}\right)$ as well as enzymatic reporters: eumelanin [also endogenous, black line:41 blue precipitate product of lacZ (blue line, ${ }^{42}$ )]; violacein (yellow line ${ }^{43}$ ). Reporter spectra were generated from published excitation spectra, normalized to the excitation maximum and multiplied by the relevant peak molar extinction coefficient. Wavelengths indicate absorption maxima. 
Table 1 Summary of the key features of different groups of RGs investigated in PA imaging.

Indirect reporters (enzymatic):

$x$ Low absorption coefficient, but...

$\checkmark$ Inherent signal amplification: one enzyme molecule can create multiple molecules of absorbing product

$\checkmark$ Not susceptible to photobleaching

Direct reporters (nonenzymatic):

$\checkmark$ Provide 1:1 mapping between the detected protein and the RG expression

- GFP-like fluorescent proteins:

$x$ Susceptible to photobleaching

$x$ Absorption maxima typically overlap with high blood attenuation, preventing deep tissue imaging

- Fluorescent proteins from phytochromes:

$x$ Typically require a cofactor (although this is not a problem in mammals)

$\checkmark$ Less susceptible to photobleaching

$\checkmark$ Allow deep tissue imaging (absorption in the NIR)

- Nonfluorescent proteins (chromoproteins):

$x$ Not yet efficiently expressed in mammalian cells

$\checkmark$ Not susceptible to photobleaching;

$\checkmark$ High PA signal generation efficiency

- Photoswitchable proteins:

$\checkmark$ Reversibly photoconvertible between red and NIR absorption states; image subtraction improves contrast, background removal, and spatial resolution

$\checkmark \quad$ NIR absorption for deep tissue imaging

$\checkmark$ No photobleaching even at fluences above the MPE

Note: NIR, near-infrared; MPE, maximum permissible exposure.

exhibit several advantages for PAI, ${ }^{60}$ such as a relatively low fluorescence quantum yield $(\sim 6 \%$ for iRFP compared to $60 \%$ for EGFP) and a high intrinsic extinction coefficient $\left(\sim 100,000 \mathrm{M}^{-1} \mathrm{~cm}^{-1}\right.$ for iRFP, compared to $\sim 56,000 \mathrm{M}^{-1} \mathrm{~cm}^{-1}$ for EGFP).

The BphP iRFP713 (also known as simply iRFP ${ }^{61}$ ) was the first to be demonstrated in PAI. iRFP expressed in tumor cells inoculated in the mammary fat pad efficiently incorporated endogenous BV, and an imaging depth of $4 \mathrm{~mm}$ was achieved exploiting the peak iRFP absorption of $690 \mathrm{~nm} .{ }^{62}$ Two subsequent studies at similar penetration depths imaged mouse brain tumors expressing iRFP713 $3^{63,64}$ and illustrated spectral resolution of two variants of iRFP (iRFP670, $645 \mathrm{~nm}$ peak and iRFP720, $702 \mathrm{~nm}^{\text {peak }}{ }^{37}$ ) as well as background hemoglobin signals. ${ }^{65}$ Despite this improved penetration depth of imaging compared to GPF-like fluorescent proteins, the photostability of BphPs remains low, due not only to photobleaching but also to transient absorption effects. ${ }^{66,67}$ Furthermore, while their low fluorescence quantum yield is attractive for PAI, fluorescent emission and ground state depopulation can lead to low PA signal generation efficiency. This may partly explain the observed discrepancies between the measured absorption and photoacoustic spectra of these proteins. ${ }^{23}$

\subsubsection{Nonfluorescent proteins (chromoproteins)}

Challenges with photobleaching and low PA signal generation has led to efforts to develop nonfluorescent (or very weakly fluorescent) proteins, also called chromoproteins [Fig. 5(b)]. Those reported to date are a subset of GFP-like proteins, which have been identified in Anthozoa species $^{31,38,39}$ or mutated from their fluorescent equivalents. ${ }^{23}$ Chromoproteins exhibit higher PA signal generation efficiency due to the absence of radiative relaxation and ground state depopulation, as well as significantly higher photostability (Fig. 8). ${ }^{23,68}$ PAI of the chromoprotein reporter ultramarine has been demonstrated in $v i v o^{68}$ in the rat ear.

\subsubsection{Photoswitchable proteins}

Photoswitchable proteins undergo a change in their absorption spectra under illumination with a specific wavelength [Fig. 5(c)]. The mechanism behind this change is typically a cis-trans isomerization of the chromophore. For the BV chromophore used in BphPs, the two conformations are referred to as Pr ("pigment red absorbing," also termed the OFF state) and Pfr ("pigment far-red absorbing," or the ON state $)^{56}$ (Fig. 10). In the unbound state, switching does not affect the absorption properties of $\mathrm{BV}$, but when covalently bound to the protein barrel the two isomeric states exhibit distinct absorption spectra. This property has been exploited in a comprehensive study of a photoswitchable protein $\mathrm{BphP} 1$ derived from the bacterial phytochrome $R p \mathrm{BphP} 1 .{ }^{19} \mathrm{By}$ taking the pixelwise subtraction between the images of the protein in the OFF and ON states, a $\sim 21$ fold enhancement in the contrast-to-noise ratio (difference between proteins and blood) was obtained relative to the $\mathrm{ON}$-state image. This differential imaging enabled visualization of BphP1-expressing U87 cells in the left kidney in PACT at depths up to $\sim 8 \mathrm{~mm}$, with an average CNR of $\sim 20$. The photoswitching property was exploited to perform subdiffraction imaging of individual cells in PAM, as BphP1 molecules are switched off at a rate proportional to the local excitation intensity, which is greater in the centre of the Gaussian-shaped laser pulse than at the periphery. In addition to this study, which illustrates the versatility of photoswitchable proteins for high contrast in vivo imaging, a preliminary demonstration has recently emerged of a second $\mathrm{BphP}$ derived photoswitchable protein, AGP1. ${ }^{22}$ Various photoswitchable proteins have also been derived from corals (GFP-like), ${ }^{69}$ two of which have been demonstrated for PAI: rsTagRFP $^{19}$ and Dronpa. ${ }^{70}$ With absorption peaks at wavelengths $<570 \mathrm{~nm}$, these proteins are most suitable for studies in PAM.

\section{Recovering the Spatial Distribution of Genetic Reporters}

Compared to fluorescence imaging of RGs, PAI can be easily performed at multiple wavelengths to specifically resolve the spatial distribution of overlapping absorption spectra. Nonetheless, achieving such spectral resolution through mathematical "unmixing" procedures is nontrivial since there are multiple endogenous absorbers present in tissue that also contribute to the signal. The position (r) and wavelength $(\lambda)$ dependent absorption coefficient $\mu_{a}(\mathbf{r}, \lambda)$ may be written as the sum of the individual molar extinction coefficients $\epsilon_{k}(\lambda)$ of the $K$ contributing chromophores with molar concentrations $c_{k}(\mathbf{r}), k=1, \ldots, K$

$\mu_{a}(\mathbf{r}, \lambda)=\sum_{k=1}^{K} c_{k}(\mathbf{r}) \epsilon_{k}(\lambda)$. 


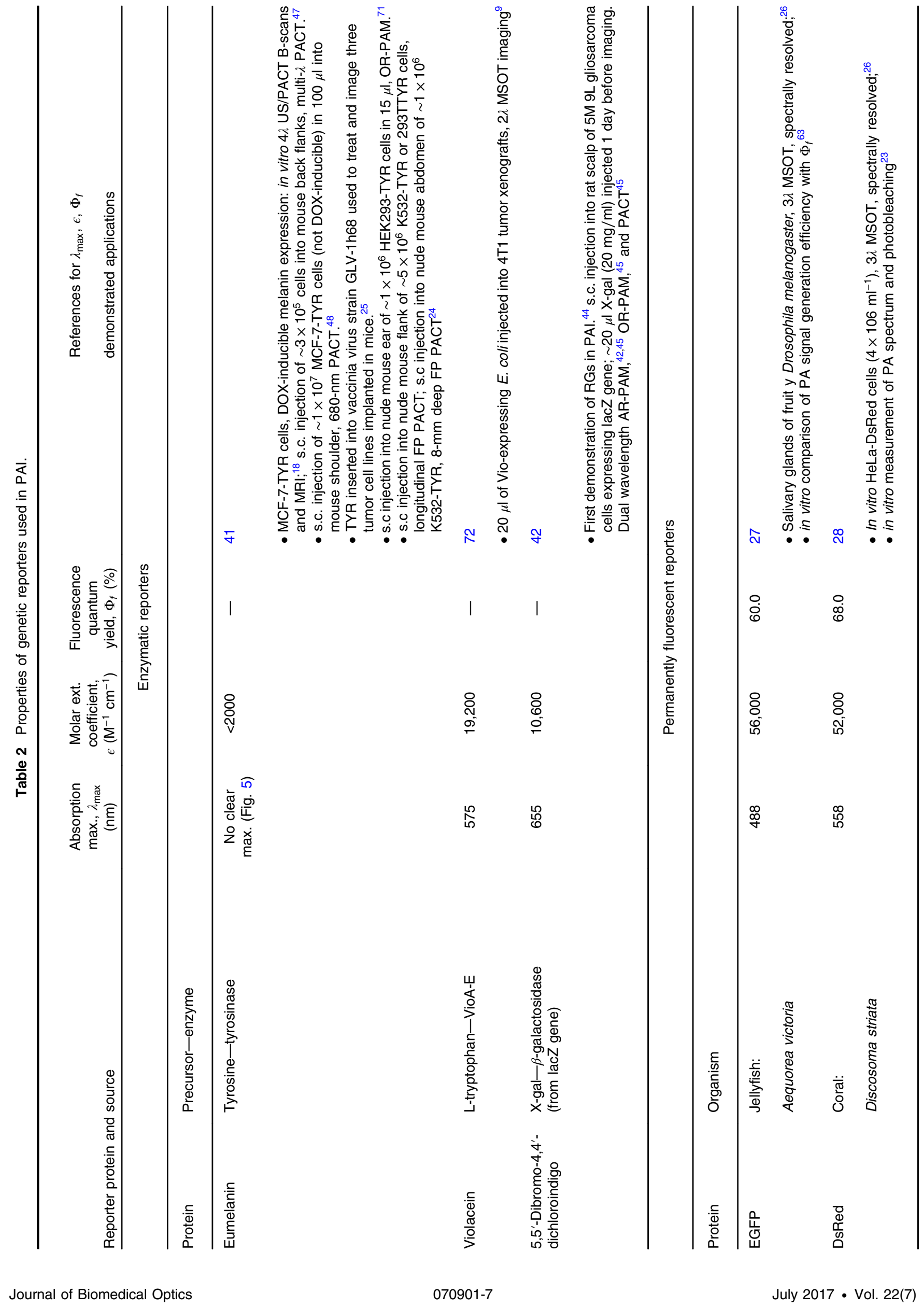




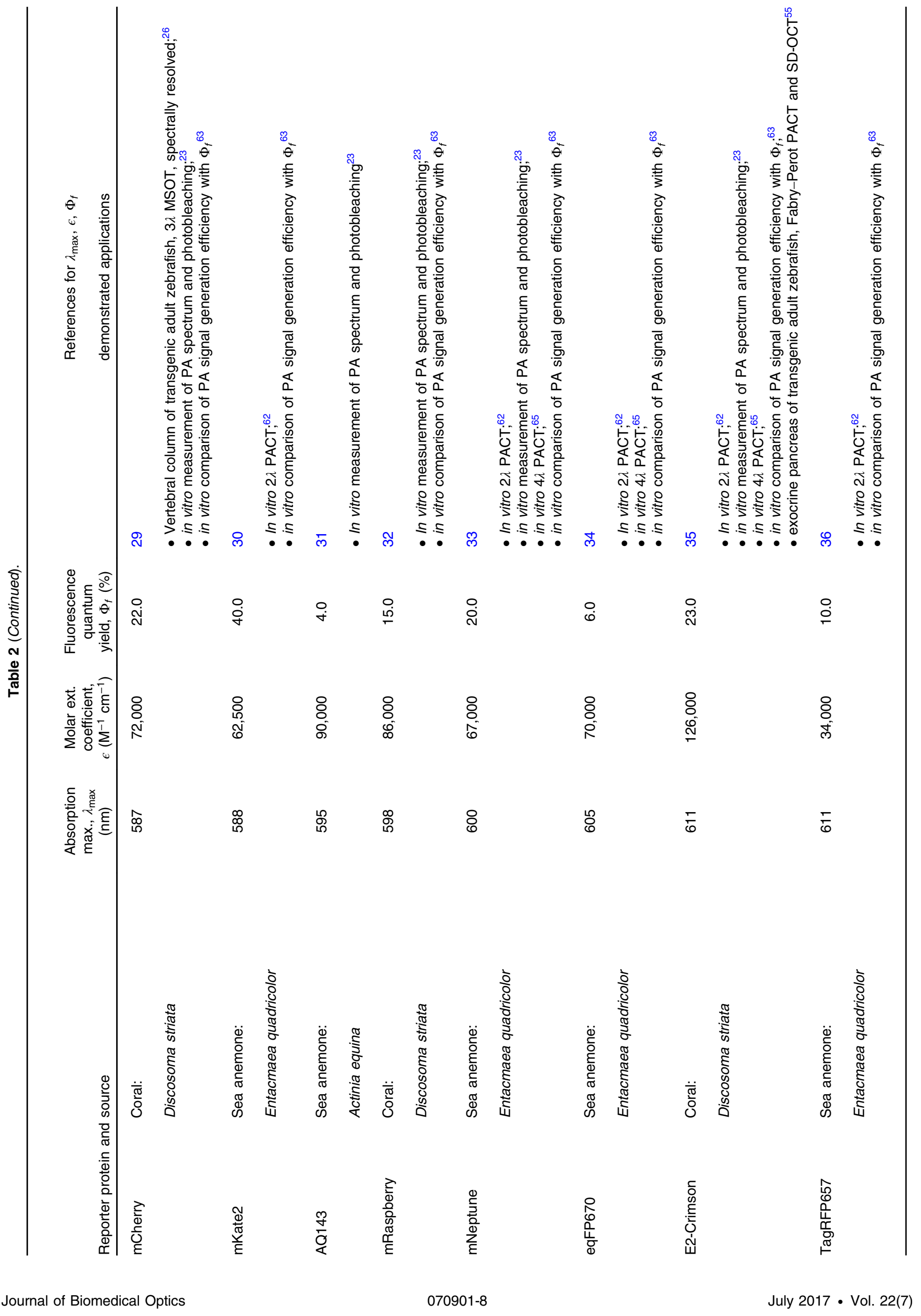


Brunker et al.: Photoacoustic imaging using genetically encoded reporters: a review

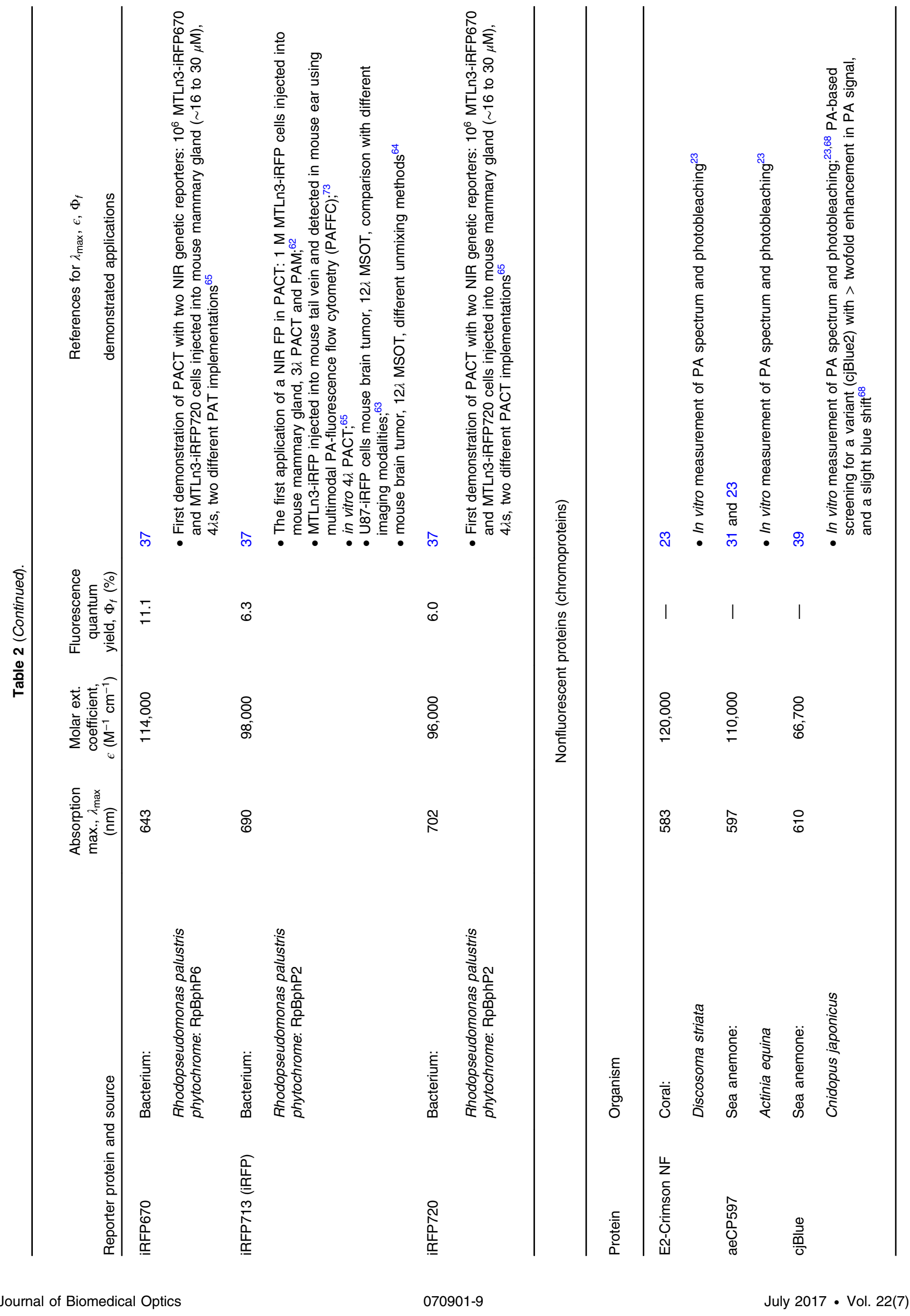


Brunker et al.: Photoacoustic imaging using genetically encoded reporters: a review

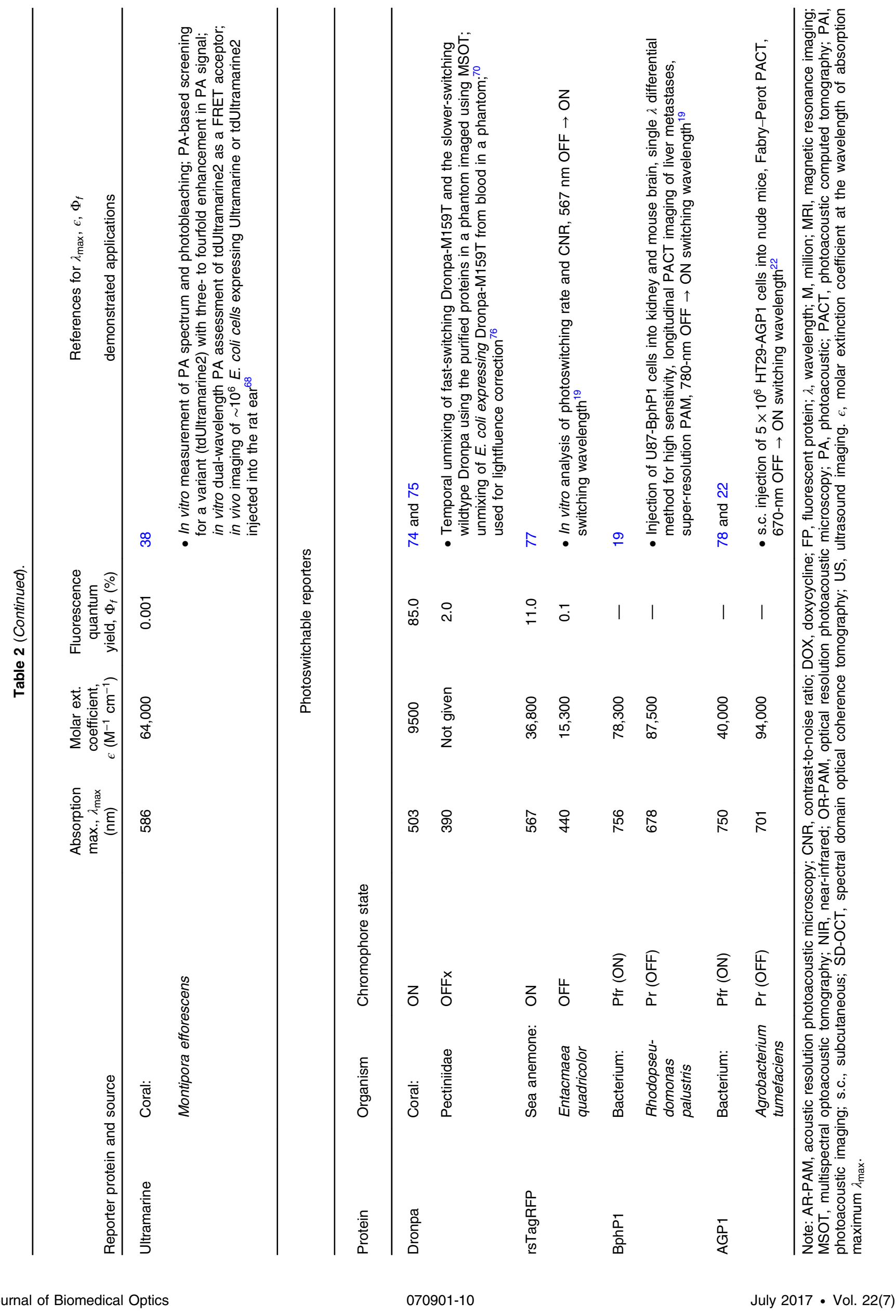


(a)

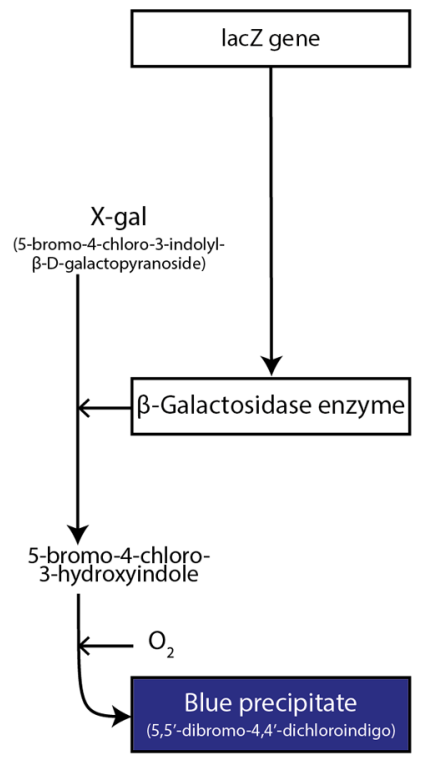

(b)

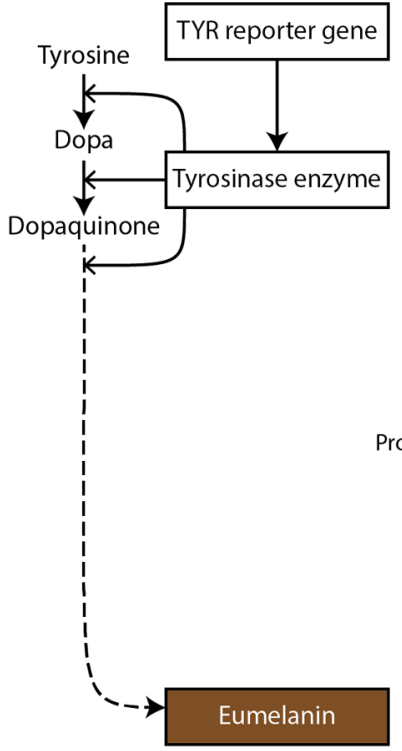

(c)

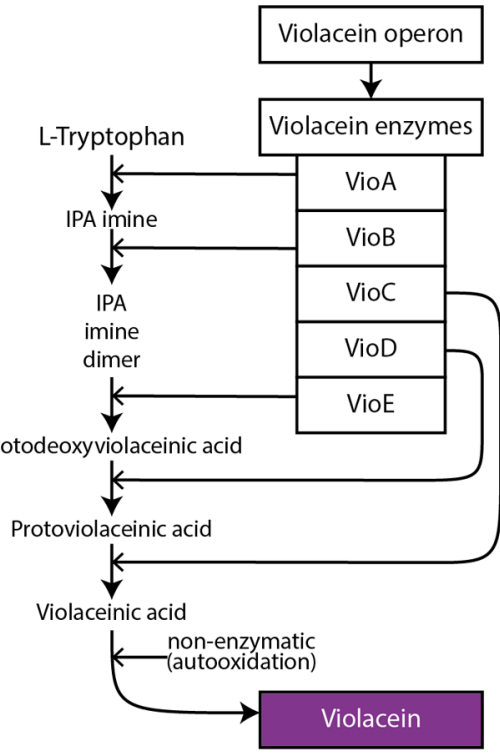

Fig. 6 Schematics showing the reaction pathways of three enzymatic reporters. (a) The hydrolysis of $\mathrm{X}$-gal catalyzed by $\beta$-galactosidase, an enzyme produced from the lacZ RG. (b) Reactions leading from tyrosine to the pigment eumelanin. The enzyme tyrosinase, produced from the tyrosinase RG, oxidizes tyrosine to dopaquinone, which then undergoes various enzymatic and nonenzymatic reactions (represented by the dashed line) leading to eumelanin. Dopa, 3,4-dihydroxyphenylalanine. (c) The violacein biosynthesis pathway. ${ }^{49}$ IPA, indole-3-pyruvic acid.

\begin{tabular}{|c|c|c|c|}
\hline Organism & Protein & Absorption (nm) & Emission $(\mathbf{n m})$ \\
\hline \multicolumn{4}{|l|}{$\begin{array}{l}\text { Animalia (Cnidaria) } \\
\text { - Anthozoa (sea anemones, corals) }\end{array}$} \\
\hline Actinia equina & $\begin{array}{l}\mathrm{AQ} 143 \\
\text { aeCP597 }\end{array}$ & $\begin{array}{l}595 \\
597\end{array}$ & $\begin{array}{c}663 \\
\text { NONE }\end{array}$ \\
\hline Cnidopus japonicus & cjBlue & 610 & NONE \\
\hline Discosoma striata & $\begin{array}{l}\text { DsRed } \\
\text { E2-Crimson-NF } \\
\text { mCherry } \\
\text { mRaspberry } \\
\text { E2-Crimson }\end{array}$ & $\begin{array}{l}558 \\
583 \\
587 \\
598 \\
611\end{array}$ & $\begin{array}{c}583 \\
\text { NONE } \\
610 \\
625 \\
646 \\
\end{array}$ \\
\hline Entacmaea quadricolor & $\begin{array}{l}\text { rsTagRFP } \\
\text { mKate2 } \\
\text { mNeptune } \\
\text { eqFP670 } \\
\text { TagRFP657 }\end{array}$ & 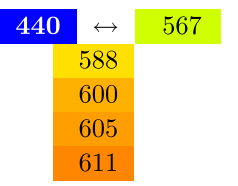 & $\begin{array}{l}585 \\
623 \\
650 \\
670 \\
657\end{array}$ \\
\hline $\begin{array}{l}\text { Montipora efflorescens } \\
\text { - Scyphozoa (jellyfish) }\end{array}$ & Ultramarine & 586 & NONE \\
\hline Aequorea victoria & EGFP & 488 & 507 \\
\hline \multicolumn{4}{|l|}{$\begin{array}{l}\text { Bacteria (Proteobacteria) } \\
\text { - Alphaproteobacteria }\end{array}$} \\
\hline $\begin{array}{r}\text { Rhodopseudomonas palustris } \\
\text { phytochrome: } R p \mathrm{BphP} 1 \\
\text { phytochrome: } R p \mathrm{BphP} 6 \\
\text { phytochrome: } R p \mathrm{BphP} 2 \\
\text { phytochrome: } R p \mathrm{BphP} 2\end{array}$ & $\begin{array}{l}\text { BphP1 } \\
\text { iRFP670 } \\
\text { iRFP713 (iRFP) } \\
\text { iRFP720 }\end{array}$ & $\begin{array}{r}\leftrightarrow \\
643 \\
690 \\
702\end{array}$ & $\begin{array}{c}\text { NONE } \\
670 \\
713 \\
720\end{array}$ \\
\hline
\end{tabular}

Fig. 7 Classification and origins of nonenzymatic fluorescent (wavelengths of absorption and emission maxima given), nonfluorescent (only absorption maxima), and photoswitchable $(\leftrightarrow)$ reporter proteins used in PAl. The colors indicate the corresponding wavelengths. Full molar extinction spectra are given in Fig. 5. 


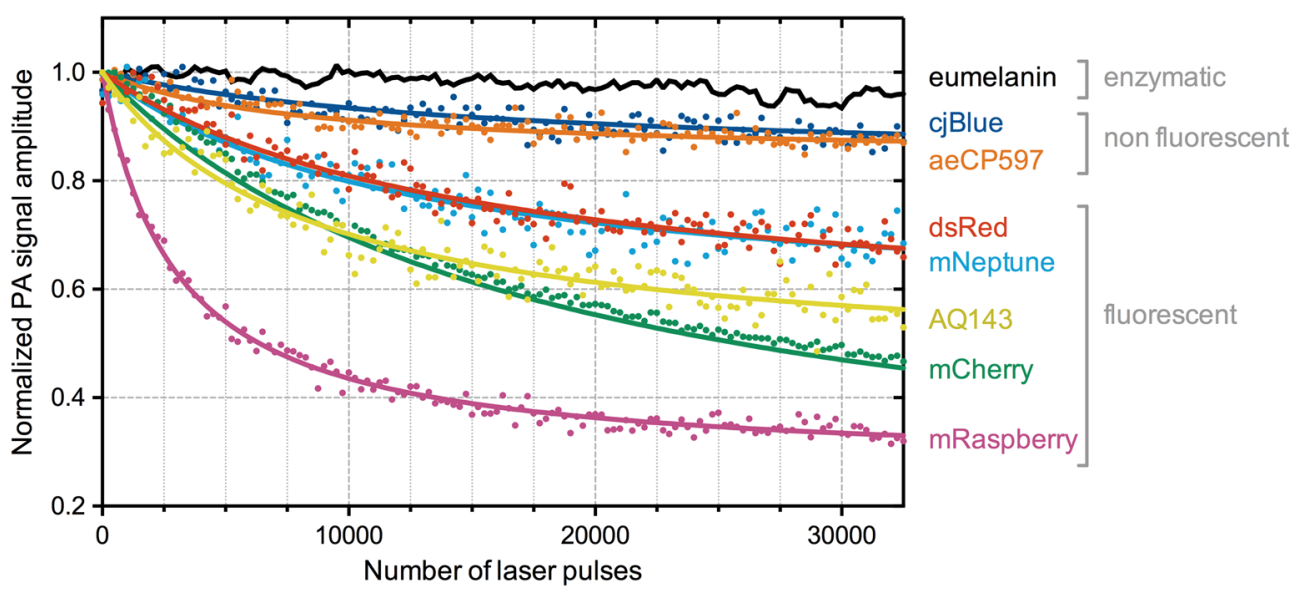

Fig. 8 Photostability of eumelanin-producing cells (the cells express the enzyme tyrosinase), compared with photobleaching of fluorescent and nonfluorescent proteins under prolonged exposure to nanosecond laser pulses. The incident fluence was in the range 1.5 to $1.7 \mathrm{~mJ} \mathrm{~cm}^{-2}$ and the pulse repetition frequency was $50 \mathrm{~Hz}$. Reproduced with permission from Refs. 23 and 24.

The spectra of $\epsilon_{k}(\lambda)$ are often known or can be measured using reference spectrophotometers, and so, in principle, it should be straightforward to recover the concentrations $c_{k}(\mathbf{r})$ from measurements of $\mu_{a}(\mathbf{r}, \lambda)$. In practice, however, the PA signal $P(\mathbf{r}, \lambda)$ is not a direct measurement of $\mu_{a}(\mathbf{r}, \lambda)$ alone; rather, it is a combination of: the optical energy $H(\mathbf{r}, \lambda)$ deposited in tissue; a proportionality constant $\alpha(\mathbf{r})$ arising due to the imperfect response of the PAI hardware used to record the acoustic data;

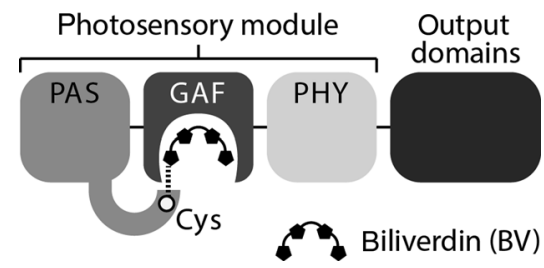

Fig. 9 Domain structure of BphPs. The photosensory module consists of PAS, GAF, and PHY domains. The chromophore BV is located in a pocket of the GAF domain and is covalently bound to the cysteine (Cys) residue of the PAS domain. Upon light absorption BV undergoes a conformational change that is detected by the photosensory module, which then transmits the signal to the effector domain, initiating the light driven molecular signaling pathway. Adapted from Ref. 56. and the Grüneisen parameter $\Gamma(\mathbf{r})$, which is a dimensionless coefficient representing the efficiency of the conversion from heat to pressure

$P(\mathbf{r}, \lambda)=\alpha(\mathbf{r}) \Gamma(\mathbf{r}) H(\mathbf{r}, \lambda)$

For in vivo imaging, the parameters $\alpha(\mathbf{r})$ and $\Gamma(\mathbf{r})$ are commonly assumed to be spatially constant (i.e., $\alpha, \Gamma$ ) and invariant with wavelength, ${ }^{47}$ giving a direct proportionality between the absorbed energy density map $H(\mathbf{r}, \lambda)$ and the detected PA signal $P(\mathbf{r}, \lambda)$. However, $H(\mathbf{r}, \lambda)$ is the product of the fluence $\Phi$, which itself varies as a function of $\mathbf{r}$ and $\lambda$, as well as $\mu_{a}(\mathbf{r}, \lambda)$ and the reduced scattering coefficient $\mu_{s}^{\prime}(\mathbf{r}, \lambda)$

$H(\mathbf{r}, \lambda)=\mu_{a}(\mathbf{r}, \lambda) \Phi\left[\mathbf{r}, \lambda, \mu_{a}(\mathbf{r}, \lambda), \mu_{s}^{\prime}(\mathbf{r}, \lambda)\right]$.

Recovery of $\mu_{a}(\mathbf{r}, \lambda)$ from Eq. (3) is an ill-posed and nonlinear inversion, in which $\Phi$ is normally unknown. Therefore, recovery of $\mu_{a}(\mathbf{r}, \lambda)$ from $P(\mathbf{r}, \lambda)$ remains a key challenge for quantitative evaluation of RG concentrations $c_{k}(\mathbf{r})$ from PA images. Nonetheless, resolving the spatial distribution and relative signal weighting for cells expressing PAI RGs may be achieved by (Table 3): (1) peak wavelength imaging; (2) spectral unmixing; or (3) difference imaging. The first approach simply maps the

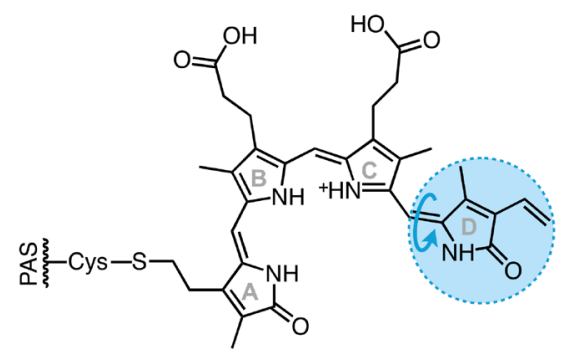

Trans conformation: Pfr state
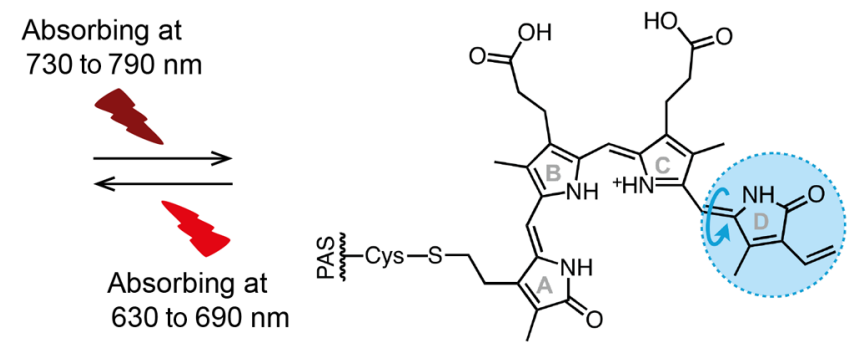

Cis conformation:

Pr state

Fig. 10 Photoswitching of the BV chromophore between the $\operatorname{Pfr}(\mathrm{ON})$ and $\operatorname{Pr}(\mathrm{OFF})$ states. Switching from ON to OFF and vice versa is induced by illumination with NIR light ( 730 to $790 \mathrm{~nm})$ and far-red light $(\sim 630$ to $690 \mathrm{~nm})$, respectively, and involves an out-of-plane rotation of the D pyrrole ring around the $C 15 / 16$ bond between the $C$ and $D$ rings. Adapted from Ref. 19. 
Table 3 Summary of methods used to separate chromophores in PA imaging.

\begin{tabular}{|c|c|c|}
\hline Method & Details, advantages, $\oplus$ and disadvantages $\ominus$ & References \\
\hline \multirow[t]{3}{*}{ Peak $\lambda$ imaging } & $\begin{array}{l}\text { Maps } H(\mathbf{r}, \lambda) \text { by thresholding signals at a } \lambda \text { where the chromophore } \\
\mu_{a} \gg \text { background absorption }\end{array}$ & $\begin{array}{l}18,24,44 \\
45,48, \text { and } 50\end{array}$ \\
\hline & $\oplus$ Simple to implement and fast to compute & \\
\hline & $\ominus$ Arbitrary threshold; signal may be lost in background & \\
\hline Linear inversion & $H(\mathbf{r}, \lambda)$ is divided by $\Phi_{\text {est }} ;$ chromophores are then separated by least squares regression & \\
\hline \multirow[t]{3}{*}{ - $\Phi_{\text {est }}$ calculated } & Assumes homogeneous background optical properties & 26 and 62 \\
\hline & $\oplus$ Simple to implement and fast to compute & \\
\hline & $\ominus$ Limited accuracy for nonsuperficial tissue layers & 79 \\
\hline \multirow[t]{5}{*}{ - $\Phi_{\text {est }}$ determined experimentally } & $\oplus$ Potential for $\Phi_{\text {est }}$ to accurately estimate $\Phi$ & \\
\hline & $\ominus$ No approach seems practical for in vivo measurements: & \\
\hline & $\rightarrow$ Black absorber inserted invasively & 80 and 81 \\
\hline & $\rightarrow$ DOT only gives a low resolution fluence map & 82 \\
\hline & $\rightarrow$ Poor speckle contrast for $\mathrm{AO}$ assisted fluence correction & 83 \\
\hline \multirow[t]{4}{*}{$\begin{array}{l}\text { - } \Phi_{\text {est }} \text { modeled and } \\
\mu_{a} \text { recovered iteratively }\end{array}$} & $\begin{array}{l}\text { Light transport model using assumed } \mu_{s}^{\prime} \text { and initialized } \mu_{a} \text { to give } \Phi_{\text {est }} \text { and } H_{\text {est }} \text {; } \\
\text { iterative optimization relative to } H(\mathbf{r}, \lambda)\end{array}$ & \\
\hline & $\begin{array}{l}\rightarrow \text { Simulations, phantoms, and in vivo studies have used the RTE, } \\
\text { or its diffuse approximation }\end{array}$ & $84-88$ \\
\hline & $\oplus$ Potential for $\Phi_{\text {est }}$ to accurately estimate $\Phi$ & \\
\hline & $\ominus$ Distribution of $\mu_{s}^{\prime}$ is often unknown in vivo & \\
\hline \multirow[t]{5}{*}{ Nonlinear model-based inversion } & $\begin{array}{l}\text { Iterative search for combinations of } \mu_{a} \text { and } \mu_{s}^{\prime} \text { that minimize the difference between } \\
H(\mathbf{r}, \lambda) \text { and } H_{\text {est }}\end{array}$ & 89 and 90 \\
\hline & $\oplus$ Little prior information required & \\
\hline & $\ominus$ Nonunique values for $\left[\mu_{a}, \mu_{s}^{\prime}\right]$ give identical $H_{\text {est }}$ & \\
\hline & requiring workarounds such as multiple illuminations & $91-93$ \\
\hline & $\ominus$ Many variables, occupying extensive computer memory & \\
\hline \multirow[t]{9}{*}{ Semiquantitative, blind unmixing } & $\begin{array}{l}\text { Use statistical properties of chromophore distributions to unmix relative contributions } \\
\text { rather than absolute } c_{k}(\mathbf{r})\end{array}$ & \\
\hline & $\rightarrow$ Vertex component analysis & 94 \\
\hline & $\rightarrow$ AMF & 95 and 96 \\
\hline & $\rightarrow \mathrm{PCA}$ & 97 \\
\hline & $\rightarrow \mathrm{ICA}$ & 63 and 98 \\
\hline & $\oplus$ Can provide more accurate results than linear inversion & \\
\hline & $\oplus$ Easy to implement and low computational cost & \\
\hline & $\ominus$ Requires correction for spectral coloring & \\
\hline & $\ominus$ Cannot estimate absolute $c_{k}(\mathbf{r})$ & \\
\hline \multirow[t]{6}{*}{ Novel decomposition methods } & Separate $\mu_{a}$ from $\Phi$ without using light transport models & \\
\hline & $\oplus$ No fluence modeling required; & \\
\hline & $\ominus$ Usually complex; not yet generally applicable in vivo & \\
\hline & $\rightarrow$ Sparse representations based on spatial characteristics & 99 \\
\hline & $\rightarrow$ Linear superposition of reference fluence base spectra & 100 \\
\hline & $\rightarrow$ "Differential" PA signal generated by two different $\lambda$ & 101 \\
\hline \multirow[t]{4}{*}{ Difference imaging } & $\begin{array}{l}\text { Pixelwise subtraction of images acquired with a photoswitchable reporter in } \\
\text { two different absorption states }\end{array}$ & $19,22,70$, and 76 \\
\hline & $\oplus$ No knowledge of the fluence required & \\
\hline & $\oplus$ Enables resolution enhancement in OR-PAM & 19 \\
\hline & $\ominus$ Only possible for photoswitchable reporters & \\
\hline
\end{tabular}

Note: AO, acousto-optics; DOT, diffuse optical tomography; RTE, radiative transport equation. $c_{k}(\mathbf{r})$, chromophore concentrations; $H(\mathbf{r}, \lambda)$, absorbed energy density; $H_{\text {est }}$, estimated $H(\mathbf{r}, \lambda) ; \lambda$, wavelength; $\mu_{a}$, absorption coefficient; $\mu_{s}^{\prime}$, reduced scattering coefficient; $\Phi_{\text {est }}$, estimated fluence. AMF (adaptive matched filter), PCA (principal component analysis), and ICA (independent component analysis). 

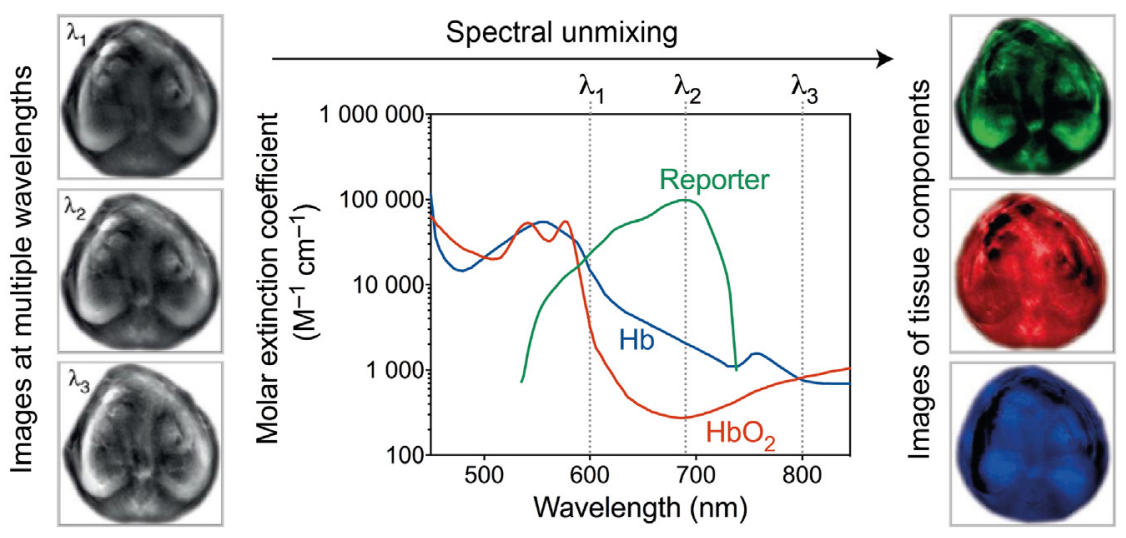

Fig. 11 Multispectral PA imaging. Images at multiple wavelengths $(\lambda)$ are combined with spectral unmixing algorithms to separate different absorbers. $\mathrm{Hb}$, deoxyhemoglobin $\mathrm{HbO}_{2}$, oxyhemoglobin reporter example, iRFP713. Adapted from Ref. 102.

spatial distribution of signals above the background at a wavelength where the absorption of the chromophore of interest is high, but the background absorption dominated by the hemoglobins is relatively low. ${ }^{18,24,44,45,48,50}$ The latter approaches are more complex, as discussed below.

\subsection{Spectral Unmixing}

Spectral unmixing takes advantage of the multiwavelength data acquisition capability of PAI, applying multivariate analysis techniques to retrieve abundance maps of the individual absorbers present in the tissue (Fig. 11). First, the aforementioned challenge of unknown light fluence $\Phi$ must be accounted for, which is usually achieved with the addition of a preprocessing step. Multiwavelength PA data are typically divided by an estimated value of the fluence $\Phi_{\text {est }}(\mathbf{r}, \lambda)$ to give images proportional to $\mu_{a}(\mathbf{r}, \lambda)$. For imaging of the superficial tissue layers in PAM, the light fluence $\Phi$ may be assumed to be spectrally constant ${ }^{71}$ and can be estimated experimentally, for example, by invasively inserting a film with uniform absorption to correct for absorption by the dermis. ${ }^{80,81}$ For applications in PACT, a simple finiteelement method solution to the light diffusion equation assuming homogeneous background optical properties can be applied to derive $\Phi_{\text {est }}(\mathbf{r}, \lambda) .{ }^{26,55,62,65}$ More rigorous approaches to deal with this "spectral coloring" in PACT include Monte Carlo simulations and model-based iterative minimization; ${ }^{84-90}$ however, these methods are computationally intensive and may give rise to nonunique solutions. As a result, they are not routinely applied during in vivo imaging.

Having dealt with the data preprocessing, most RG studies perform linear inversion using a form of least-squares regression, ${ }^{26,55,62,65}$ in some cases with constraints for positive concentration values exceeding some threshold. ${ }^{47,68}$ These least-squares approaches rely on a priori knowledge of the absorption spectra present within a given voxel. Where these are not well known, for example, when the spectra change as a function of concentration, semiquantitative "blind" methods that use the inherent statistical properties of the spectral data can be employed. Some examples relevant to RG imaging include: principle component analysis (PCA) $;{ }^{97}$ independent component analysis (ICA); $; 3,98$ vertex component analysis; ${ }^{94}$ and adaptive matched filter (AMF). ${ }^{64,95}$ Only $\mathrm{AMF}^{64}$ and $\mathrm{ICA}^{63}$ have been applied in PAI of RGs; both methods were able to resolve brain tumors in mice expressing iRFP713. Tzoumas et al. ${ }^{64}$ assessed the impact of the number of excitation wavelengths and different unmixing algorithms on the detection sensitivity for the RG, although their findings contradict prior studies suggesting that the optimum number of wavelengths and unmixing algorithm is likely to be case sensitive. ${ }^{95}$

\subsection{Difference Imaging}

Spectral unmixing is hampered by fundamental requirements: the preprocessing must be accurate to compensate for light fluence, and the data must have sufficient signal-to-noise ratio (SNR) to avoid corruption of the spectral profile. The recent emergence of photoswitchable nonfluorescent proteins [Fig. 4(c), Sec. 3.4 $]^{19,22,70}$ mitigates these limitations by switching the protein ON/OFF state between image acquisitions. Taking the difference of images acquired at the same wavelength but with different protein states effectively eliminates the nonswitchable background signals, minimizing the effect of the local optical fluence. ${ }^{76}$ As a result, photoswitchable reporters achieve reliable background removal and have been shown to dramatically enhance the detection sensitivity ( $\sim 34$-fold enhancement in contrast-to-noise ratio compared to twowavelength least-squares fitting ${ }^{19}$ ). For applications requiring quantification of protein concentration, rather than only qualitative spatial localization, photoswitchable reporters therefore represent an ideal solution.

\section{Detection Sensitivity}

The ability to detect and resolve RGs in vivo relies on sufficient sensitivity in the PAI hardware, as well as biological considerations, such as gene expression levels and spectral characteristics of the reporter.

\subsection{Imaging System Sensitivity}

For molecular PAI, spatial resolution and penetration depth have to be traded to optimize the detection sensitivity. One parameter used to quantify detection sensitivity is the noise-equivalent detectable concentration (NEC) ${ }^{79}$ NEC is calculated as the concentration of the imaged molecules normalized by the SNR. To achieve a low NEC, one can increase the excitation light fluence (within the maximum permissible exposure, MPE) to increase the SNR. Since the SNR of PAI is proportional to the total number of molecules in a resolution voxel, relaxing the spatial 
resolution can also improve the NEC, as long as the local optical fluence is maintained. Counter-intuitively, this means that PACT systems, which generally have worse spatial resolution, typically have a better NEC than PAM systems, provided that the light pulse energy is sufficient.

The detection sensitivity of PAI is generally lower than that of pure optical imaging modalities by at least two orders of magnitude. ${ }^{79}$ This is somewhat surprising considering that, at the source (the imaged target), the overall energy conversion efficiencies of fluorescence and PA imaging are similar: for a fluorescent absorber, the transfer of energy from the absorbed photons to fluorescent emission is typically less than $30 \%$ efficient, determined by the fluorescent quantum yield, and the remaining absorbed optical energy is deposited as thermal energy, which is subsequently converted to acoustic energy with an efficiency of $\sim 20 \%$ at body temperature, determined by the Grüneisen coefficient. ${ }^{82}$ The difference in sensitivity between the two imaging modalities is largely a consequence of the detectors. While a photomultiplier tube used in fluorescence imaging may have single-photon sensitivity, a typical ultrasonic transducer has a noise-equivalent pressure (NEP) of $\sim 2 \mathrm{~Pa},{ }^{83,91}$ which is equivalent to a temperature rise at the target of $\sim 2 \mu \mathrm{K}$. Within the skin MPE $\left(20 \mathrm{~mJ} / \mathrm{cm}^{2}\right)$, the noise equivalent absorption coefficient $\left(\mathrm{NE} \mu_{a}\right)$ of the target is $\sim 0.001 \mathrm{~cm}^{-1}$. For EGFP with a molar extinction coefficient of $56,000 \mathrm{M}^{-1} \mathrm{~cm}^{-1}$ and quantum yield of $60 \%$ at $488 \mathrm{~nm}$, this $\mathrm{NE} \mu_{a}$ roughly translates to an NEC of $0.1 \mu \mathrm{M}$. When the geometric signal loss from the target to the ultrasonic transducer is taken into account, ${ }^{91,92}$ the NEC approaches several micromolars, which is generally consistent with experimental results and much higher than that of native proteins in cells. ${ }^{79}$ By contrast, the detection sensitivity of fluorescence microscopy is on the level of several nanomolars, close to the natural concentrations of native cell components.

Fundamental limitations on recorded PA signals are: PA detection sensitivity; PA signal generation efficiency; and the tissue optical and acoustic attenuation. Fortunately, the acoustic sensitivity of ultrasound detectors can be enhanced by better matching their detection bandwidth with the broadband PA signal spectrum. While the detection sensitivity of a conventional piezoelectric ultrasonic transducer degrades with decreasing element size, the sensitivity of optical sensors of acoustic waves, typically based on interferometry, does not. Therefore, optical detection holds promise for high-sensitivity PACT, especially for the cases where the elements of the ultrasonic transducer array are miniaturized to achieve isotropic resolutions. ${ }^{93,96,99}$

\subsection{Reporter Detectability}

For PAI at depths more than $1 \mathrm{~mm}$, the reporter protein should have an absorption maximum within the tissue optical window (620 to $950 \mathrm{~nm})$ where mammalian tissues are relatively transparent due to the low optical absorption by water, lipids, hemoglobin, and melanin. Even with such optimization of the reporter protein absorption wavelength, the strong background signal from blood still presents a challenge for PA RG imaging, since hemoglobin concentration in tissue is $\sim 2.3 \mathrm{mM}{ }^{100}$ The problem is shown in Figs. 5(d) and 5(e), which compares the molar extinction spectra and specific extinction spectra for the hemoglobins and a selection of enzymatic and direct PAI reporters. The molecular mass of enzymatically produced proteins is more than two orders of magnitude smaller than the molecular mass of the hemoglobins and directly produced proteins, such as mCherry or BphP1. Thus, care must be taken when comparing required reporter concentrations in terms of moles, grams, or gene-expressing cell numbers. As an example of the challenges of reporter detectability, while the absorption maximum of $\mathrm{BphP} 1$ is in the tissue optical window, if the protein concentration is two orders of magnitude lower than hemoglobin concentrations, it remains a challenge to detect a signal above the hemoglobin background. Nonetheless, proteins derived from bacterial phytochromes (iRFP670 and iRFP720) have been detected at estimated concentrations in vivo of 16 to $30 \mu \mathrm{M},{ }^{65}$ although the effect of such high concentrations on cell biology remains unknown.

\section{Potential Applications}

RGs for PAI have the potential to be applied in a variety of fundamental biomedical studies that require high spatial resolution at extended penetration depths. Examples include but are not limited to: (1) visualizing the expression of genes of interest; (2) understanding signal transduction pathways and proteinprotein interactions; ${ }^{101}$ (3) longitudinal monitoring of tumor growth, ${ }^{24,19}$ (4) tracking the in vivo distribution of administered cells (e.g., therapeutic immune cells); (5) probing distancedependent interactions between two proteins (Förster resonance energy transfer); ${ }^{68}$ and (6) reporting on environment change due to biochemical activities (e.g., calcium ion concentration). Furthermore, complementing functional PAI of neuronal activities in deep brain (>2 mm) using hemoglobin signals, ${ }^{103}$ novel genetic reporters of action potentials or surrogates (e.g., voltage-, or calcium-sensitive proteins) would be of great advantage $^{104}$ and could potentially be engineered from nonfluorescent near-infrared BphPs. ${ }^{105}$ An additional future area of interest could be development of PAI optogenetic sensors to modulate neuronal activities in deep brain, where the lightmediated control of protein-protein interactions could be monitored at high resolution. ${ }^{106}$

\section{Challenges and Prospects}

PAI has filled a void of high-resolution optical imaging in deep tissue, opening the potential for biomedical studies that require high-resolution RG imaging at depths beyond the optical diffusion limit. Moreover, the use of nonionizing radiation in PAI allows longitudinal molecular imaging of the same animal, enabling informative observations of disease progression or drug efficacy. The properties of existing reporters are compared in Table 1. Chromoproteins are promising given their high PA signal generation efficiency but have not yet been expressed efficiently in mammalian cells. So far, despite requiring tailored instrumentation, photoswitchable proteins show the greatest potential for deep tissue in vivo PA imaging, since differential imaging between ON/OFF states removes the endogenous background signal improving image contrast and SNR.

The major challenges in PAI of RGs are sensitivity and quantification. The detection sensitivity is ultimately limited by the instrumentation but can be somewhat mitigated by choosing reporters with favorable absorption characteristics and high protein expressions levels. Recovering the spatial distribution of reporters has been approached using two distinct methods. Spectral unmixing results in large uncertainties and false positives, mainly due to the unknown light fluence. Using photoswitchable phytochromes is potentially only limited by 
detection noise. Both approaches remain susceptible to motion artifacts, which can be overcome by minimizing the time interval between image acquisitions. ${ }^{107-109}$ The focus for future studies should be to optimize detection sensitivity and to improve reporter localization and quantification. This requires technical improvements in acoustic transducer sensitivity, and addressing signal unmixing challenges such as the unknown light fluence. Further biological improvements could develop and optimize the spectra and extinction coefficients of photoswitchable reporters applied in difference imaging.

The number of RGs for PAI has expanded dramatically in recent years with examples of high-resolution and highsensitivity detection of reporter proteins now available. Future developments in PAI hardware and protein engineering will push the current detection limits further, enabling PAI to emerge as a modality of choice for longitudinal molecular imaging of genetic reporters.

\section{Disclosures}

The authors have no relevant financial interests in this article and no other potential conflicts of interest to disclose.

\section{Acknowledgments}

The authors would like to thank Amit Jathoul for helpful discussion. J.B. and S.E.B. are supported by the EPSRCCRUK Cancer Imaging Centre in Cambridge and Manchester (No. C197/A16465); CRUK (Nos. C14303/A17197 and C47594/A16267); and the European Union's Seventh Framework Programme (No. FP7/2007-2013) under Grant Agreement No. FP7-PEOPLE-2013-CIG-630729. J.Y. is partly supported by Duke MEDx Basic Research Grant. J.L. acknowledges the support of ERC Starting Grant No. 281356.

\section{References}

1. R. Weissleder and V. Ntziachristos, "Shedding light onto live molecular targets," Nat. Med. 9(1), 123-128 (2003).

2. C. Martelli et al., "Optical imaging probes in oncology," Oncotarget 7(30), 48753-48787 (2016).

3. J. Weber, P. C. Beard, and S. E. Bohndiek, "Contrast agents for molecular photoacoustic imaging," Nat. Methods 13, 639-650 (2016).

4. M. L. James and S. S. Gambhir, "A molecular imaging primer: modalities, imaging agents, and applications," Physiol. Rev. 92(2), 897-965 (2012).

5. H. Youn and J. K. Chung, "Reporter gene imaging," Am. J. Roentgenol. 201(2), W206-W214 (2013).

6. C. Liu et al., "Advances in imaging techniques and genetically encoded probes for photoacoustic imaging," Theranostics 6(13), 2414-2430 (2016).

7. C. Lutzweiler and D. Razansky, "Optoacoustic imaging and tomography: reconstruction approaches and outstanding challenges in image performance and quantification," Sensors 13, 7345-7384 (2013).

8. K. P. Koestli et al., "Temporal backward projection of optoacoustic pressure transients using Fourier transform methods," Phys. Med. Biol. 46, 1863-1872 (2001).

9. M. $\mathrm{Xu}$ and L. V. Wang, "Universal back-projection algorithm for photoacoustic computed tomography," Phys. Rev. E 71, 016706 (2005).

10. A. Rosenthal, D. Razansky, and V. Ntziachristos, "Fast semi-analytical model-based acoustic inversion for quantitative optoacoustic tomography," IEEE Trans. Med. Imaging 29, 1275-1285 (2010).

11. G. Paltauf et al., "Iterative reconstruction algorithm for optoacoustic imaging," J. Acoust. Soc. Am. 112(4), 1536-1544 (2002).

12. B. E. Treeby and B. T. Cox, "k-Wave: MATLAB toolbox for the simulation and reconstruction of photoacoustic wave fields," J. Biomed. Opt. 15(2), 021314 (2010).
13. B. E. Treeby, J. Jaros, and B. T. Cox, "Advanced photoacoustic image reconstruction using the k-Wave toolbox," Proc. SPIE 9708, 97082P (2016).

14. B. Cox et al., "Quantitative spectroscopic photoacoustic imaging: a review," J. Biomed. Opt. 17(6), 061202 (2012).

15. L. V. Wang and S. Hu, "Photoacoustic tomography: in vivo imaging from organelles to organs," Science 335, 1458-1462 (2012).

16. L. V. Wang and J. Yao, "A practical guide to photoacoustic tomography in the life sciences," Nat. Methods 13(8), 627-638 (2016).

17. T. K. Kim and J. H. Eberwine, "Mammalian cell transfection: the present and the future," Anal. Bioanal. Chem. 397(8), 3173-3178 (2010).

18. R. J. Paproski et al., "Tyrosinase as a dual reporter gene for both photoacoustic and magnetic resonance imaging," Biomed. Opt. Express 2, 771-780 (2011).

19. J. Yao et al., "Multiscale photoacoustic tomography using reversibly switchable bacterial phytochrome as a near-infrared photochromic probe," Nat. Methods 13, 67-73 (2016).

20. J. Märk et al., "Development of tyrosinase-based reporter genes for preclinical photoacoustic imaging of mesenchymal stem cells," Proc. SPIE 8943, $89433 Z$ (2014).

21. T. Dull et al., "A third-generation lentivirus vector with a conditional packaging system," J. Virol. 72(11), 8463-8471 (1998).

22. H. Dortay et al., "Dual-wavelength photoacoustic imaging of a photoswitchable reporter protein," Proc. SPIE 9708, 970820 (2016).

23. J. Laufer et al., "In vitro characterization of genetically expressed absorbing proteins using photoacoustic spectroscopy," Biomed. Opt. Express 4, 2477-2490 (2013).

24. A. P. Jathoul et al., "Deep in vivo photoacoustic imaging of mammalian tissues using a tyrosinase-based genetic reporter," Nat. Photonics 9, 239-246 (2015).

25. J. Stritzker et al., "Vaccinia virus-mediated melanin production allows MR and optoacoustic deep tissue imaging and laser-induced thermotherapy of cancer," Proc. Natl. Acad. Sci. U. S. A. 110, 3316-3320 (2013).

26. D. Razansky et al., "Multispectral opto-acoustic tomography of deepseated fluorescent proteins in vivo," Nat. Photonics 3, 412-417 (2009).

27. N. C. Shaner, P. A. Steinbach, and R. Y. Tsien, "A guide to choosing fluorescent proteins," Nat. Methods 2, 905-909 (2005).

28. B. J. Bevis and B. S. Glick, "Rapidly maturing variants of the Discosoma red fluorescent protein (DsRed)," Nat. Biotechnol. 20, 83-87 (2002).

29. N. C. Shaner et al., "Improved monomeric red, orange and yellow fluorescent proteins derived from Discosoma sp. red fluorescent protein," Nat. Biotechnol. 22, 1567-1572 (2004).

30. D. Shcherbo et al., "Far-red fluorescent tags for protein imaging in living tissues," Biochem. J 418, 567-574 (2009).

31. M. A. Shkrob et al., "Far-red fluorescent proteins evolved from a blue chromoprotein from Actinia equina," Biochem. J. 392, 649-654 (2005).

32. L. Wang et al., "Evolution of new nonantibody proteins via iterative somatic hypermutation," Proc. Natl. Acad. Sci. U. S. A. 101, 1674516749 (2004).

33. M. Z. Lin et al., "Autofluorescent proteins with excitation in the optical window for intravital imaging in mammals," Chem. Biol. 16, 11691179 (2009).

34. D. Shcherbo et al., "Near-infrared fluorescent proteins," Nat. Methods 7, 827-829 (2010).

35. R. L. Strack et al., "A rapidly maturing far-red derivative of DsRedExpress2 for whole-cell labeling," Biochemistry 48, 8279-8281 (2009).

36. K. S. Morozova et al., "Far-red fluorescent protein excitable with red lasers for flow cytometry and superresolution STED nanoscopy," Biophys. J. 99(2), L13-L15 (2010).

37. D. M. Shcherbakova and V. V. Verkhusha, "Near-infrared fluorescent proteins for multicolor in vivo imaging," Nat. Methods 10, 751-754 (2013).

38. A. Pettikiriarachchi et al., "Ultramarine, a chromoprotein acceptor for Förster resonance energy transfer," PLoS One 7, e41028 (2012).

39. M. C. Y. Chan et al., "Structural characterization of a blue chromoprotein and its yellow mutant from the sea anemone Cnidopus japonicus," J. Biol. Chem. 281, 37813-37819 (2006).

40. S. Prahl, "Tabulated molar extinction coefficient for hemoglobin in water," http://omlc.org/spectra/hemoglobin/summary.html (1998).

41. T. Sarna and H. M. Swartz, "Extinction coefficient of melanin," http://omlc.org/spectra/melanin/extcoeff.html (1988). 
42. L. Li et al., "Simultaneous imaging of a lacZ-marked tumor and microvasculature morphology in vivo by dual-wavelength photoacoustic microscopy," J. Innovative Opt. Health Sci. 1(2), 207-215 (2008).

43. R. S. Blosser and K. M. Gray, "Extraction of violacein from Chromobacterium violaceum provides a new quantitative bioassay for N-acyl homoserine lactone autoinducers," J. Microbiol. Methods 40(1), 47-55 (2000).

44. L. Li et al., "Photoacoustic imaging of lacZ gene expression in vivo," J. Biomed. Opt. 12(2), 020504 (2007).

45. X. Cai et al., "Multi-scale molecular photoacoustic tomography of gene expression," PLoS One 7, e43999 (2012).

46. I. Braasch, M. Schartl, and J.-N. Volff, "Evolution of pigment synthesis pathways by gene and genome duplication in fish," BMC Evol. Biol. 7(1), 74 (2007).

47. R. J. Paproski et al., "Multi-wavelength photoacoustic imaging of inducible tyrosinase reporter gene expression in xenograft tumors," Sci. Rep. 4, 5329 (2014).

48. C. Qin et al., "Tyrosinase as a multifunctional reporter gene for photoacoustic/MRI/PET triple modality molecular imaging," Sci. Rep. 3, 1490 (2013).

49. C. Dantas, R. Tauler, and M. M. C. Ferreira, "Exploring in vivo violacein biosynthesis by application of multivariate curve resolution on fused UV-VIS absorption, fluorescence, and liquid chromatographymass spectrometry data," Anal. Bioanal. Chem. 405(4), 1293-1302 (2013).

50. Y. Jiang et al., "Violacein as a genetically-controlled, enzymatically amplified and photobleaching-resistant chromophore for optoacoustic bacterial imaging," Sci. Rep. 5, 11048 (2015).

51. G. U. Nienhaus and J. Wiedenmann, "Structure, dynamics and optical properties of fluorescent proteins: perspectives for marker development," ChemPhysChem 10(9-10), 1369-1379 (2009).

52. J. Wiedenmann, F. Oswald, and G. U. Nienhaus, "Fluorescent proteins for live cell imaging: opportunities, limitations, and challenges," IUBMB Life 61(11), 1029-1042 (2009).

53. O. V. Stepanenko et al., "Beta-barrel scaffold of fluorescent proteins," Int. Rev. Cell Mol. Biol. 302, 221-278 (2013).

54. G. Zhang, V. Gurtu, and S. R. Kain, "An enhanced green fluorescent protein allows sensitive detection of gene transfer in mammalian cells," Biochem. Biophys. Res. Commun. 227, 707-711 (1996).

55. M. Liu et al., "In vivo three dimensional dual wavelength photoacoustic tomography imaging of the far red fluorescent protein E2-Crimson expressed in adult zebrafish," Biomed. Opt. Express 4, 1846-1855 (2013).

56. D. M. Shcherbakova, M. Baloban, and V. V. Verkhusha, "Near-infrared fluorescent proteins engineered from bacterial phytochromes," Curr. Opin. Chem. Biol. 27, 52-63 (2015).

57. P. A. Steinbach and R. Y. Tsien, "Mammalian expression of infrared fluorescent proteins engineered from a bacterial phytochrome," Science 324, 804-807 (2009).

58. D. Yu et al., "An improved monomeric infrared fluorescent protein for neuronal and tumour brain imaging," Nat. Commun. 5, 3626 (2014).

59. D. Yu et al., "A naturally monomeric infrared fluorescent protein for protein labeling in vivo," Nat. Methods 12, 763-765 (2015).

60. K. D. Piatkevich, F. V. Subach, and V. V. Verkhusha, "Engineering of bacterial phytochromes for near-infrared imaging, sensing, and lightcontrol in mammals," Chem. Soc. Rev. 42(8), 3441-3452 (2013).

61. G. S. Filonov et al., "Bright and stable near-infrared fluorescent protein for in vivo imaging," Nat. Biotechnol. 29, 757-761 (2011).

62. G. S. Filonov et al., "Deep-tissue photoacoustic tomography of a genetically encoded near-infrared fluorescent probe," Angew. Chem. Int. Ed. 51, 1448-1451 (2012).

63. N. C. Deliolanis et al., "Deep-tissue reporter-gene imaging with fluorescence and optoacoustic tomography: a performance overview," Mol. Imaging Biol. 16, 652-660 (2014).

64. S. Tzoumas et al., "Effects of multispectral excitation on the sensitivity of molecular optoacoustic imaging," J. Biophotonics 8, 629-637 (2015).

65. A. Krumholz et al., "Multicontrast photoacoustic in vivo imaging using near-infrared fluorescent proteins," Sci. Rep. 4, 3939 (2014).

66. R. B. Vegh et al., "Chromophore photoreduction in red fluorescent proteins is responsible for bleaching and phototoxicity," J. Phys. Chem. B 118(17), 4527-4534 (2014).
67. J. Zhu et al., "Ultrafast excited-state dynamics and fluorescence deactivation of near-infrared fluorescent proteins engineered from bacteriophytochromes," Sci. Rep. 5, 12840 (2015).

68. Y. Li et al., "Engineering dark chromoprotein reporters for photoacoustic microscopy and FRET imaging," Sci. Rep. 6, 22129 (2016).

69. A. C. Stiel et al., "Generation of monomeric reversibly switchable red fluorescent proteins for far-field fluorescence nanoscopy," Biophys. J. 95(6), 2989-2997 (2008).

70. A. C. Stiel et al., "High-contrast imaging of reversibly switchable fluorescent proteins via temporally unmixed multispectral optoacoustic tomography," Opt. Lett. 40, 367 (2015).

71. A. Krumholz et al., "Photoacoustic microscopy of tyrosinase reporter gene in vivo," J. Biomed. Opt. 16(8), 080503 (2011).

72. A. S. Mendes et al., "Factorial design and response surface optimization of crude violacein for Chromobacterium violaceum production," Biotechnol. Lett. 23(23), 1963-1969 (2001).

73. D. A. Nedosekin et al., "Synergy of photoacoustic and fluorescence flow cytometry of circulating cells with negative and positive contrasts," J. Biophotonics 6(5), 425-434 (2013).

74. R. Ando, H. Mizuno, and A. Miyawaki, "Regulated fast nucleocytoplasmic shuttling observed by reversible protein highlighting," Science 306(5700), 1370-1373 (2004).

75. S. Habuchi et al., "Reversible single-molecule photoswitching in the GFP-like fluorescent protein Dronpa," Proc. Natl. Acad. Sci. U.S.A. 102(27), 9511-9516 (2005).

76. X. L. Dean-Ben et al., "Light fluence normalization in turbid tissues via temporally unmixed multispectral optoacoustic tomography," Opt. Lett. 40(20), 4691 (2015).

77. F. V. Subach et al., "Red fluorescent protein with reversibly photoswitchable absorbance for photochromic FRET," Chem. Biol. 17(7), 745-755 (2010).

78. T. Lamparter et al., "Phytochrome from Agrobacterium tumefaciens has unusual spectral properties and reveals an N-terminal chromophore attachment site," Proc. Natl. Acad. Sci. U.S.A. 99(18), 11628-11633 (2002).

79. R. Hochuli, P. C. Beard, and B. Cox, "Accuracy of approximate inversion schemes in quantitative photacoustic imaging," Proc. SPIE, Photons Plus Ultrasound: Imaging and Sensing 8943, 89435V (2014).

80. K. Maslov, H. F. Zhang, and L. V. Wang, "Effects of wavelengthdependent fluence attenuation on the noninvasive photoacoustic imaging of hemoglobin oxygen saturation in subcutaneous vasculature in vivo," Inverse Prob. 23, S113-S122 (2007).

81. H. F. Zhang et al., "Imaging of hemoglobin oxygen saturation variations in single vessels in vivo using photoacoustic microscopy," Appl. Phys. Lett. 90(5), 053901 (2007).

82. A. Q. Bauer et al., "Quantitative photoacoustic imaging: correcting for heterogeneous light fluence distributions using diffuse optical tomography," J. Biomed. Opt. 16(9), 096016 (2011).

83. A. Hussain et al., "Quantitative blood oxygen saturation imaging using combined photoacoustics and acousto-optics," Opt. Lett. 41(8), 1720-1723 (2016).

84. B. Cox et al., "Two-dimensional quantitative photoacoustic image reconstruction of absorption distributions in scattering media by use of a simple iterative method," Appl. Opt. 45(8), 1866-1875 (2006).

85. Z. Yuan and H. Jiang, "Quantitative photoacoustic tomography: recovery of optical absorption coefficient maps of heterogeneous media," Appl. Phys. Lett. 88(23), 231101 (2006).

86. L. Yao, Y. Sun, and H. Jiang, "Quantitative photoacoustic tomography based on the radiative transfer equation," Opt. Lett. 34, 1765-1767 (2009).

87. Y. Sun and H. Jiang, "Quantitative three-dimensional photoacoustic tomography of the finger joints: phantom studies in a spherical scanning geometry," Phys. Med. Biol. 54, 5457-5467 (2009).

88. F. M. Brochu et al., "Towards quantitative evaluation of tissue absorption coefficients using light fluence correction in optoacoustic tomography," IEEE Trans. Med. Imaging 36, 322-331 (2017).

89. B. T. Cox, S. R. Arridge, and P. C. Beard, "Estimating chromophore distributions from multiwavelength photoacoustic images," J. Opt. Soc. Am. A 26(2), 443-455 (2009).

90. J. Laufer et al., "Quantitative determination of chromophore concentrations from 2D photoacoustic images using a nonlinear model-based inversion scheme," Appl. Opt. 49, 1219-1233 (2010). 
91. R. J. Zemp, "Quantitative photoacoustic tomography with multiple optical sources," Appl. Opt. 49(18), 3566-3572 (2010).

92. G. Bal and K. Ren, "Multi-source quantitative photoacoustic tomography in a diffusive regime," Inverse Prob. 27(7), 075003 (2011).

93. P. Shao, B. Cox, and R. J. Zemp, "Estimating optical absorption, scattering, and Grueneisen distributions with multiple-illumination photoacoustic tomography," Appl. Opt. 50(19), 3145-3154 (2011).

94. X. L. Deán-Ben et al., "Fast unmixing of multispectral optoacoustic data with vertex component analysis," Opt. Lasers Eng. 58, 119-125 (2014).

95. S. Tzoumas et al., "Unmixing molecular agents from absorbing tissue in multispectral optoacoustic tomography," IEEE Trans. Med. Imaging 33(1), 48-60 (2014).

96. S. Tzoumas, "Statistical molecular target detection framework for multispectral optoacoustic tomography," IEEE Trans. Med. Imaging 35(12), 2534-2545 (2016).

97. J. Glatz et al., "Blind source unmixing in multi-spectral optoacoustic tomography," Opt. Express 19, 3175-3184 (2011).

98. X. L. Deán-Ben et al., "Estimation of optoacoustic contrast agent concentration with self-calibration blind logarithmic unmixing," Phys. Med. Biol. 59, 4785-4797 (2014).

99. A. Rosenthal, D. Razansky, and V. Ntziachristos, "Quantitative optoacoustic signal extraction using sparse signal representation," IEEE Trans. Med. Imaging 28(12), 1997-2006 (2009).

100. S. Tzoumas et al., "Eigenspectra optoacoustic tomography achieves quantitative blood oxygenation imaging deep in tissues," Nat. Commun. 7, 12121 (2016).

101. S. S. Choi et al., "Wavelength-Modulated Differential Photoacoustic Spectroscopy (WM-DPAS) for noninvasive early cancer detection and tissue hypoxia monitoring," J. Biophotonics 9(4), 388-395 (2016).

102. A. Taruttis and V. Ntziachristos, "Advances in real-time multispectral optoacoustic imaging and its applications," Nat. Photonics 9, 219-227 (2015).

103. X. Wang et al., "Noninvasive laser-induced photoacoustic tomography for structural and functional in vivo imaging of the brain," Nat. Biotechnol. 21, 803-806 (2003).

104. A. Devor et al., "The challenge of connecting the dots in the B.R.A.I. N.," Neuron 80(2), 270-274 (2013).

105. D. M. Shcherbakova et al., "Natural photoreceptors as a source of fluorescent proteins, biosensors, and optogenetic tools," Ann. Rev. Biochem. 84, 519-550 (2015).

106. A. A. Kaberniuk, A. A. Shemetov, and V. V. Verkhusha, "A bacterial phytochrome-based optogenetic system controllable with near-infrared light," Nat. Methods 13, 591-597 (2016).
107. X. L. Deán-Ben, E. Bay, and D. Razansky, "Functional optoacoustic imaging of moving objects using microsecond-delay acquisition of multispectral three-dimensional tomographic data," Sci. Rep. 4, 5878 (2014).

108. J. Märk et al., "Photoacoustic pump-probe tomography of fluorophores in vivo using interleaved image acquisition for motion suppression," Sci. Rep. 7, 40496 (2017).

109. L. Li et al., "Single-impulse panoramic photoacoustic computed tomography of small-animal whole-body dynamics at high spatiotemporal resolution," Nat. Biomed. Eng. 1, 0071 (2017).

Joanna Brunker received her MSc degree in natural sciences and her $\mathrm{PhD}$ in medical physics and biomedical engineering from the University College London, UK. She is a postdoctoral research fellow at the Cancer Research UK Cambridge Institute. Her research interest is the engineering of reporters and the development of instrumentation for functional photoacoustic imaging, with a focus on tumor hypoxia.

Junjie J. Yao received his BE and ME degrees from Tsinghua University and his $\mathrm{PhD}$ in biomedical engineering from Washington University, St. Louis. $\mathrm{He}$ is an assistant professor of biomedical engineering at Duke University and a faculty member of Duke Center for In Vivo Microscopy and Fitzpatrick Institute for Photonics. His research interest is in photoacoustic tomography technologies in life sciences, especially in functional brain imaging and early cancer detection.

Jan Laufer graduated as a diplom-ingenieur in biomedical engineering from the University of Applied Sciences Lbeck and received his $\mathrm{PhD}$ in medical physics from the University College London, UK. $\mathrm{He}$ is a professor of medical physics at the Martin-Luther-Universitä Halle-Wittenberg, Germany. His research interest is the development of methods and technologies for molecular and functional photoacoustic imaging.

Sarah E. Bohndiek received her BA degree from the University of Cambridge and her $\mathrm{PhD}$ in radiation physics from the University College London. She is a group leader in biomedical physics at the University of Cambridge. Her research is focused on the development of innovative optical imaging techniques to enable earlier diagnosis of cancer. 